\title{
Monopoly models with time-varying demand function
}

\author{
Fausto Cavallia,*, Ahmad Naimzada ${ }^{\mathrm{b}}$ \\ ${ }^{a}$ Department of Mathematical Sciences, Mathematical Finance and Econometrics, Catholic \\ University of the Sacred Heart, Via Necchi 9, 20123 Milano, Italy \\ ${ }^{b}$ Department of Economics, Management and Statistics, University of Milano-Bicocca, \\ Milan, Italy
}

\begin{abstract}
We study a family of monopoly models for markets characterized by timevarying demand functions, in which a boundedly rational agent chooses output levels on the basis of a gradient adjustment mechanism. After presenting the model for a generic framework, we analytically study the case of cyclically alternating demand functions. We show that both the perturbation size and the agent's reactivity to profitability variation signals can have counterintuitive roles on the resulting period- 2 cycles and on their stability. In particular, increasing the perturbation size can have both a destabilizing and a stabilizing effect on the resulting dynamics. Moreover, in contrast with the case of timeconstant demand functions, the agent's reactivity is not just destabilizing, but can improve stability, too. This means that a less cautious behavior can provide better performance, both with respect to stability and to achieved profits. We show that, even if the decision mechanism is very simple and is not able to always provide the optimal production decisions, achieved profits are very close to those optimal. Finally, we show that in agreement with the existing empirical literature, the price series obtained simulating the proposed model exhibit a significant deviation from normality and large volatility, in particular when underlying deterministic dynamics become unstable and complex.
\end{abstract}

Keywords: Time-varying demand, Monopoly model, Bounded rationality, Gradient mechanism, Complex dynamics

\section{Introduction}

The debate about economic agents' rationality plays a central role in economic research. As Baumol and Quandt [1] remarks, "since all real decisions are made under conditions of imperfect information", it is more sensible to assume that agents try to "learn" the economic context in which they operate, in order

\footnotetext{
* Corresponding author

Email addresses: fausto.cavalli@unicatt.it (Fausto Cavalli), ahmad.naimzada@unimib.it (Ahmad Naimzada)
} 
to optimize their performance. This, which is indeed true for complex economic settings, is valid for very simple markets, too, as for example monopolies, in which the complete knowledge of the demand by the agent can be doomed, as noticed in [2], by "...lagged response of buyers to changing prices, exogenous changes in tastes, techniques, government policies...", and the demand curve can change "its slope, being highly elastic for a stretch, then perhaps becoming relatively inelastic" (see [3]).

In particular, the monopolistic setting appears to be a very useful prototypical benchmark to study the modelling of simple and feasible learning techniques. Clower in [2] proposed several ways to describe how an "ignorant monopolist", with different informational endowments, can replace the goal of finding the profit maximizing output level or price with the goal of dynamically approximating it, in a sort of try-go wrong-learn process. This can be achieved by using a rule-of-thumb technique ([1]), by which, starting from objectively measurable economic variables, the agent can take simple and suitably inexpensive decisions. For example, a typical family of rule-of-thumbs is based on the information provided by the variation of the achieved profits. If, as a consequence of increasing production levels or prices, profits increase, then a naive rule consists in keeping on increasing them. Conversely, if profits decrease, the agent would more likely move in the opposite direction, trying to decrease the output level or the price. From the mathematical point of view, such adjustment mechanism can be realized by imposing that decisions change proportionally to the (continuous or discrete) gradient of the profit function, where the proportionality constant represents the agent's reactivity to profitability change signals. Gradient mechanisms are used to model several economic contexts characterized by boundedly rational agents. As an example, starting from the contribution by Bischi and Naimzada in [4], such kind of mechanism has been applied to the modelling of oligopoly competitions $([5,6,7,8,9])$. Concerning the monopoly modelling, we can mention the contribution by Puu [10], who studied the chaotic dynamics arising when a discrete gradient is considered, while Naimzada and Ricchiuti [11] investigated the effect of the continuous gradient. In both works a discrete time adjustment mechanism is considered. Subsequent contributions extended the investigations in several directions, taking into account different demand and cost functions $[12,13,14,15]$, studying the effect of varying the price elasticity of demand [16], considering modelling approaches based on continuous differential equations $[17,18]$ or on hybrid discrete-continuous equations [19]. For a survey about monopoly modelling, we refer to [20]

However, an issue, already raised in $[2,1]$, has never been studied. What happens when the demand and/or the cost functions are variable in time? Is again possible, for an boundedly rational monopolistic agent, to learn the profit maximizing production/price decision by adopting a rule-of-thumb like those considered for constant in time demand/cost functions? This is exactly the aim of the present contribution, in which we study a monopolistic firm which adapts its production decisions on the basis of local information about the profit function in an economic context characterized by a time-varying demand function. After describing how the gradient mechanism can be adapted to such a kind of 
market, we study a simple situation, in which a demand function periodically shifts. This choice is motivated by the research perspective proposed by Baumol and Quandt in [1, p. 41]. Such theoretical model is intrinsically founded on the real example of goods whose request is affected by seasonality, as for instance energy ([21]), household furniture, clothing, toys, food ([22, 23, 24]). We remark that in such a more complicated market setting, it is even more reasonable to assume that the agent is unable to have a perfect knowledge of the demand, and must rely on simple mechanisms. The model we present is based on a non-autonomous discrete difference equation. Periodically perturbed dynamical systems have been investigated from the mathematical point of view and applied to scientific modelling: in both cases, the literature is very wide. Just limiting to mathematical investigations, we mention the contributions by Grinfeld et al. [25], in which the periodically perturbed logistic equation is studied, by AlSharawi et al. [26], in which Sharkovsky's theorem is generalized to periodically perturbed equations and by Elaydi et al. [27], in which the authors develop the local bifurcation theory for one-dimensional difference equations. In classical monopoly models based on the gradient approach, the agent's reaction has no effect on the possible steady states of dynamical model. This means that if the optimum production level is a steady state of the dynamical model for some values of the parameter $\gamma$ representing the agent's reaction speed, then this is true for any $\gamma$. Moreover, parameter $\gamma$ has an unambiguous destabilizing role, as increasing $\gamma$ can just lead a stable steady state to lose stability through a flip bifurcation.

We have several consequences of taking into account a periodic perturbation. Firstly, the model is no more represented through an autonomous difference equation, like in the classical situation, but it is intrinsically non-autonomous. Moreover, the optimal production level of the unperturbed monopoly is necessarily replaced by a couple of optimal production levels, each one maximizing profits obtained with the corresponding perturbed demand function. As a consequence, the resulting perturbed dynamical model based on a gradient adjustment mechanism does not posses a steady state, but its simplest attractor is a period-2 cycle. We prove and show that the introduction of a shifting demand function has remarkable effects on the the role of the agent's reaction speed on the production trajectories and on the stability of dynamics. In fact, the agent's reaction speed affects the period- 2 cycles of the perturbed model, which, in general, do not consist of the two optimal production decisions. Moreover, with respect to the stability, the role of $\gamma$ is much more ambiguous, since, besides the classical destabilizing role, increasing $\gamma$ can be stabilizing too. The role of the perturbation size is counterintuitive, too. In fact, considering a demand function with an inflection point, we show that introducing a perturbation and increasing its size can indeed have a destabilizing effect, leading stable dynamics to chaotic ones, but it can even lead chaotic unperturbed dynamics to become stable.

We stress that in the adjustment mechanism we study, the agent never uses any information about the underlying actual time variability of the demand. It is reasonable to ask if such a mechanism is too unsophisticated, as the agent 
should become aware of nature of the demand and since the mechanism is not able to let the agent "learn" the profit maximizing output levels. Firstly, we prove that even under such reduced information endowment, the agent is able to learn (provided that stability holds) the qualitative period-2 cyclicity of the demand function. In particular, using the correct profitability variation signal coming from the estimate of the marginal profit function, the output level, for any $\gamma$, is increased when the market size is large and decreased when it is small. Moreover, even if period-2 cycles do not coincide with profit maximizing output levels, the actual achieved profits are very close to the optimal profits. In any case, even in the simple deterministic framework characterized by alternating demands, the superimposition of non-deterministic effects (due for instance to climate shocks, consumer's preferences' variability,...) can make more difficult for the agent to use the precise demand function variability. The mechanism we studied, which requires a very reduced rationality level, is then suitably effective to result reliable, showing at the same time how the agent's behavior in a complex economic environment can lead to complex, sometimes counterintuitive effects. This is also confirmed by the agreement, in the qualitative aspects, between simulated time series and empiric data. In fact simulated price series exhibit the non-normal distribution, asymmetry and large volatility of real price series of seasonal goods ([28]). In particular, we show that the joint effect of a nonlinear economic setting affected by a deterministic oscillation, a boundedly rational mechanism for the economic agent and the presence of small superimposed random fluctuations are essential for the emergence of the above mentioned stylized facts.

The remainder of the paper is organized as follows. In Section 2 we introduce the monopoly model; in Section 3 we analytically study the cyclically perturbed problem; in Section 4 we present several simulative investigations; in Section 5 we investigate qualitative properties of simulated price series; in Section 6 we summarize achieved results and we propose possible research perspectives. Appendix collects Propositions' proofs.

\section{Monopoly models for time-varying demand functions}

In the setting we assume the monopolistic firm has the market power and decides the output level $q>0$ of the good it produces. In what follows, we focus on strictly positive production levels, and we implicitly assume that the equilibria of any optimization problem we consider are strictly positive. When a dynamical adjustment process is present, we also restrict to starting production levels that give rise to output trajectories that never cross economically uninteresting or unfeasible regions (as those in which $q \leq 0$ ).

Our main assumption is that the market we study is characterized by a timevarying demand curve. Before introducing the model, we firstly summarize, as a reference situation, the classical model in which the demand function is constant over time. To this end, we consider the inverse demand function $p_{0}: I_{0} \rightarrow \mathbb{R}^{+}$, where $I_{0} \subset(0,+\infty)$. Since the focus of this work concerns the time-varying demand function, we consider for the monopolistic firm the very simple form of 
cost function $c(q)=c q$, i.e. we assume constant marginal costs. This, which is the most common assumption in the related literature, also allows us to disentangle the effects of the variable demand from possible other sources of complexity.

Consistently with [2] we assume an "ignorant" monopolist, who, endowed with a reduced information setting about the economic environment and/or limited computational capabilities, adopts a "rule of thumb". In the present work, as in [11], we consider a monopolist who, in order to choose production level $q_{t+1}$ at time $t+1$, adapts the quantity $q_{t}$ produced at time $t$ proportionally to the variation of profits, namely to the gradient of the profit function. More details about this mechanism and its economic interpretation can be found in $[16,19]$ and in the references therein. In the classical monopoly market based on such approach, it is assumed that by market researches the monopolist is able to obtain the correct slope of the profit function when the produced quantity lies in a neighborhood of $q_{t}$, so that the marginal profit function is locally known. The resulting monopoly model is then represented by the onedimensional autonomous equation

$$
q_{t+1}=q_{t}+\gamma \pi_{0}^{\prime}\left(q_{t}\right),
$$

where $\gamma>0$ represents the reactivity (or adjustment speed) of the agent and $\pi_{0}^{\prime}\left(q_{t}\right)=p_{0}(q)+q p_{0}^{\prime}(q)-c$ is the marginal profit function corresponding to profit function $\pi_{0}(q)=q p_{0}(q)-c q$.

If the market is characterized by a price function which varies over time, the previous mechanism may be modified in several ways. If we assume that the agent is completely aware of the deterministic variability of the demand function over time, then a possibility is that, in order to decide the production level $q_{t+1}$, the agent uses information about prices and profits coming a time $t+1-n$ at which the market was characterized by the same (or at least very similar) demand function of the next period $t+1$, maybe taking into account also information from times $t-n+2, \ldots, t$ adopting some kind of average. However, this assumption strictly relies on an elevated rationality degree for the agent, who must have a perfect knowledge of the underlying deterministic tendency of the demand. We notice that the resulting model would be significantly more refined and complex than (1). On the other hand, we can assume that the agent completely neglects that the demand function can vary over time and uses only information from time $t$. In practice, the monopolist, just after the market realization at time $t$, tries to obtain the correct estimation of the marginal profits in a neighborhood of the production level $q_{t}$. However, this setting encompasses a too reduced rationality degree for the agent. Actually, the profitability change signal obtained at time $t$ can be opposite with respect to that at time $t+1$. For instance, we analytically and numerically checked that this can lead to decrease the production level even if the demand will increase. Then, it is more reasonable that the agent tries to obtain the correct estimation of the marginal profits near the production level $q_{t}$ just before the next production decision, namely approaching time $t+1$, when such information is more reliable for the 
next period production decision. Such framework does not require that the agent knows the precise kind of variability of the demand: he/she just assume that the demand can change over time and so tries to collect information as close as possible to the production decision. This is particularly suitable if, for instance, we suppose that "...a sufficiently long interval of time had elapsed" between $t$ and $t+1$ "to permit the monopolist to discover the new position and properties of the demand function" [2].

On this basis, we can assume that if the inverse demand is described by a function $p_{t}(q)$ which varies over time, the resulting model is

$$
q_{t+1}=q_{t}+\gamma \pi_{t+1}^{\prime}\left(q_{t}\right)
$$

where $\pi_{t+1}^{\prime}\left(q_{t}\right)=p_{t+1}\left(q_{t}\right)+q_{t} p_{t+1}^{\prime}\left(q_{t}\right)-c$ is the marginal profit function. We notice that even if we assumed that the agent does not know the precise underlying variability of the demand function, he/she uses the correct profitability (variation) signal. We remark that in model (2) both the learning process and the production decisions occur at the discrete time level $\{t\}$. We can indeed assume that the demand function varies on an underlying continuous time level $\tau$, namely that it is represented by a time-continuous function $p(q, \tau)$. Model $(2)$ then represents the situation in which both the new learning process and the production decision at time period $t+1$ occurs after a suitably large amount of time, during which the agent can realize that the demand changed and then can try to collect information about it as time the new production decision for time period $t+1$ approaches.

In what follows, we assume that both time-constant and time-varying demand functions, as well as marginal costs, are such that the respective profit functions have a unique maximum attained for some strictly positive output level. This can be realized by imposing that marginal profit functions fulfill first order conditions

$$
\pi_{0}^{\prime}\left(q^{*}\right)=0, \quad \pi_{t}^{\prime}\left(q_{t}^{*}\right)=0, t>0
$$

respectively at the unique positive output level $q^{*}>0$ and at the sequence of positive output levels $q_{t}^{*}>0$, and that all the profit functions are strictly concave, for which it is sufficient to assume

$$
\pi_{0}^{\prime \prime}(q)<0, \quad \pi_{t}^{\prime \prime}(q)<0
$$

for any $t>0$. Then both $q^{*}$ and $q_{t}^{*}, t \geq 0$ are global maximum points of the respective profit functions, and they then coincide with the optimal production levels of markets respectively characterized by inverse demand functions $p_{0}(q)$ and $p_{t}(q)$. We notice that the actual evolution of $q_{t}^{*}$ strictly depends on how the demand function $p_{t}(q)$ varies over time and that in a monopoly characterized by a time-varying demand function, the resulting model is intrinsically nonautonomous. 


\section{Analysis}

In the previous Section we introduced model (2) in a quite general economic setting. In this Section we want to focus on a simple scenario, which, at the same time, allows us to consider an economically significant situation and to keep the modelling framework analytically tractable. To this end, we consider periodically perturbed demand functions, in which the perturbation consists in vertical, possibly asymmetrical shifts of the time-constant demand function $p_{0}$. From now on, we will refer to $p_{0}$ as the unperturbed inverse demand function. In particular, we consider the simplest kind of cyclicity, so that the market is characterized by two alternating inverse demand functions $p_{1}(q)=p_{0}(q)+h_{1}(\varepsilon)$ and $p_{2}(q)=p_{0}(q)+h_{2}(\varepsilon)$, where functions $h_{i}$ represent the perturbation depending on the perturbation size $\varepsilon$. We assume that $h_{1}$ (resp. $h_{2}$ ) is a strictly increasing (resp. strictly decreasing) differentiable function defined on some interval $[0, \hat{h}) \subseteq \mathbb{R}^{+}$, satisfying $h_{1}(0)=0\left(\right.$ resp. $h_{2}(0)=0$ ), which then provides a positive, upward (resp. negative, downward) shift of $p_{0}$. We have that when $\varepsilon=0$ we are actually considering the unperturbed demand function, while as $\varepsilon$ increases, the perturbation extent increases, too. With such kind of perturbation, we have that if the same price is considered, when the market is characterized by $p_{1}$ the demanded quantity is larger than that with the unperturbed demand $p_{0}$, which, in turn, provides a larger demanded quantity than $p_{2}$. We notice that either both positive or both negative perturbations of $p_{0}$ can be considered as well, but this does not provide significantly different results from those reported in this section and in Section 4. Finally, without loss of generality, we assume that the market is characterized by the inverse demand function $p_{1}$ (respectively $p_{2}$ ) when $t$ is odd (respectively even), so that we can introduce function

$$
\xi_{t}(\varepsilon)= \begin{cases}h_{1}(\varepsilon) & \text { if } t \text { is odd } \\ h_{2}(\varepsilon) & \text { if } t \text { is even, }\end{cases}
$$

and rewrite the general profit function $p_{t}(q)$ as

$$
p_{t}(q)=p_{0}(q)+\xi_{t}(\varepsilon) .
$$

We can then introduce the two profit functions $\pi_{i}(q)=q p_{i}(q)-c q, i=1,2$, and, recalling the expression of $\pi_{0}^{\prime}$, we can write the corresponding marginal profit functions as

$$
\pi_{1}^{\prime}(q)=\pi_{0}^{\prime}(q)+h_{1}(\varepsilon), \quad \pi_{2}^{\prime}(q)=\pi_{0}^{\prime}(q)+h_{2}(\varepsilon) .
$$

In what follows, we will refer to $\pi_{0}$ and $\pi_{i}$ respectively as unperturbed and perturbed profit functions, and to $\pi_{0}^{\prime}$ and $\pi_{i}^{\prime}$ as unperturbed and perturbed marginal profit functions, respectively. Assumption (3a) simplifies as

$$
\pi_{0}^{\prime}\left(q_{1}^{*}\right)+h_{1}(\varepsilon)=0, \quad \pi_{0}^{\prime}\left(q_{2}^{*}\right)+h_{2}(\varepsilon)=0,
$$

in which each equation is respectively solved by the unique, positive output level $q_{i}^{*}>0, i=1,2$, while the second order condition $(3 \mathrm{~b})$ reduces to

$$
\pi_{0}^{\prime \prime}(q)<0
$$


since $\pi_{0}^{\prime \prime}=\pi_{1}^{\prime \prime}=\pi_{2}^{\prime \prime}$. We then have that $q_{i}^{*}, i=1,2$ are the optimal production levels of markets respectively characterized by inverse demand functions $p_{1}$ and $p_{2}$ and the non-autonomous model (2) can be rewritten as

$q_{t+1}=q_{t}+\gamma\left(\pi_{0}^{\prime}\left(q_{t}\right)+\xi_{t+1}\left(q_{t}\right)\right)= \begin{cases}f_{1}\left(q_{t}\right)=q_{t}+\gamma\left(\pi_{0}^{\prime}\left(q_{t}\right)+h_{1}(\varepsilon)\right) & \text { if } t+1 \text { is odd, } \\ f_{2}\left(q_{t}\right)=q_{t}+\gamma\left(\pi_{0}^{\prime}\left(q_{t}\right)+h_{2}(\varepsilon)\right) & \text { if } t+1 \text { is even. }\end{cases}$

In what follows, we assume $\gamma \in(0,1]$, that allows us to avoid that instability phenomena be introduced or strengthened by an agent's over-reaction to the profit variation signal.

For the analytical investigation of (5), in order to apply the classical theory for autonomous problems, we can introduce variables $q_{1, t}$ and $q_{2, t}$, and system

$$
\left\{\begin{array}{l}
q_{1, t+1}=g_{1}\left(q_{2, t}\right)=q_{2, t}+\gamma\left(\pi_{0}^{\prime}\left(q_{2, t}\right)+h_{1}(\varepsilon)\right) \\
q_{2, t+1}=g_{2}\left(q_{2, t}\right)=q_{2, t}+\gamma\left(\pi_{0}^{\prime}\left(q_{2, t}\right)+h_{1}(\varepsilon)\right)+\gamma\left(\pi_{0}^{\prime}\left(q_{2, t}+\gamma\left(\pi^{\prime}\left(q_{2, t}\right)+h_{1}(\varepsilon)\right)\right)+h_{2}(\varepsilon)\right) .
\end{array}\right.
$$

If we set $q_{2,0}=q_{0}$, it is possible to prove that there is a correspondence between the trajectories generated by equation (5) and by System (6), since a direct check shows that $\left(q_{1, t}, q_{2, t}\right)=\left(q_{2 t-1}, q_{2 t}\right)$ for any $t \geq 1$.

In the remaining part of this section we analytically investigate model (5) and we compare the results for the perturbed model (5) to those about the unperturbed one (1) and the underlying optimization problems (respectively concerning the unperturbed and perturbed demand functions). To simplify the explanation and to focus on the role of the parameters, propositions will be formulated abstracting from the economic context. In particular, we consider a general function $p_{0}(q)$ defined on $\mathbb{R}$, so that the domains of $p_{1}(q)$ and $p_{2}(q)$ are $\mathbb{R}$, too. Every result of this Section is still valid even if we consider functions $p_{i}$ on smaller sets. When suitable, we precise the effect of considering domains which are feasible with respect to an economic problem.

Before investigating dynamical model (5), we recall that, thanks to assumptions (4) on the profit functions, we know that output level $q^{*}>0$ is the only optimal production decision when the inverse demand function is $p_{0}$, while $q_{1}^{*}$ and $q_{2}^{*}$ are the optimal output levels when the inverse demand functions are $p_{0}+h_{1}(\varepsilon)$ and $p_{0}+h_{2}(\varepsilon)$, respectively. It is evident that $q_{i}^{*}$ are somehow connected to $q^{*}$, since as $\varepsilon_{i} \rightarrow 0^{+}$we have that the perturbed demand functions become the unperturbed one. We have the following intuitive result.

Proposition 1. Let $\pi_{0}^{\prime}: \mathbb{R} \rightarrow \mathbb{R}$ be a differentiable function satisfying (4). Then there are $\varepsilon_{M} \in(0,+\infty]$ and two continuously differentiable functions $q_{i}^{*}$ : $\left[0, \varepsilon_{M}\right) \rightarrow \mathbb{R}$ with $i=1,2$ such that $q_{i}^{*}(0)=q^{*}$. Moreover, we have $\left(q_{1}^{*}\right)^{\prime}>0$ and $\left(q_{2}^{*}\right)^{\prime}<0$.

Proposition 1 is very predictable and simply shows that under the effect of $h_{1}$ and $h_{2}$ the optimal production level $q^{*}$ is replaced, at least for suitably small values of $\varepsilon$, by the couple of output levels $q_{1}^{*}(\varepsilon)$ and $q_{2}^{*}(\varepsilon)$, which are the 
profit maximizing production levels with respect to perturbed demand functions $p_{0}(q)+h_{1}(\varepsilon)$ and $p_{0}(q)+h_{2}(\varepsilon)$, respectively. This allows us to follow, in some sense, the evolution of $q^{*}$ as the perturbation size increases. The remainder of this section is devoted to the study of period-2 cycles of model (5), in particular highlighting the effects of the perturbation size $\varepsilon$ and of the agent's reaction speed $\gamma$. In what follows, we will denote such invariant sets by $\left\{\hat{q}_{1}, \hat{q}_{2}\right\}$. In those situations in which we want to focus on the effect of changing one of the two main parameters, leaving the remaining one fixed, we use notation $\hat{q}_{i}^{a}(b)$, meaning that parameter $a$ is kept constant while $b$ is varied.

We can then wonder how the results of Proposition 1 change when a dynamical adjustment process based on a gradient mechanism like that in (5) is taken into account. Indeed, as widely shown in the literature $([11,16])$ it is straightforward to see that the profit optimizing output level $q^{*}$ of the unperturbed demand function is a steady state of the unperturbed model (1). Conversely, due to the cyclical nature of the non-autonomous equation (5), we can not expect that (5) has a steady state. The simplest invariant sets we can look for are then period-2 cycles. In particular, we can ask if, just like in Proposition 1, for any given value $\gamma$ of the reaction speed, the steady state $q^{*}$ of the unperturbed model (1) is replaced, under the effect of the perturbation, by a period-2 cycle of the perturbed model (5) and if such cycle consists of the alternation of $q_{1}^{*}$ and $q_{2}^{*}$. These issues are investigated in the following Proposition.

Proposition 2. Let $\pi_{0}^{\prime}: \mathbb{R} \rightarrow \mathbb{R}$ be a differentiable function satisfying (4). Then, for any $\gamma \in(0,1], \gamma \neq-2 / \pi_{0}^{\prime \prime}\left(q^{*}\right)$ there are $\tilde{\varepsilon}(\gamma)>0$ and two differentiable functions $\hat{q}_{i}^{\gamma}:[0, \tilde{\varepsilon}(\gamma)) \rightarrow \mathbb{R}$ for $i=1,2$ such that

(1) $\left\{\hat{q}_{1}^{\gamma}(\varepsilon), \hat{q}_{2}^{\gamma}(\varepsilon)\right\}$ is a period-2 cycle of System (5) for any $\varepsilon \in[0, \tilde{\varepsilon}(\gamma))$;

(2) $\lim _{\varepsilon \rightarrow 0^{+}} \hat{q}_{i}^{\gamma}(\varepsilon)=q^{*}$.

Proposition $2^{1}$ shows that a steady state of the unperturbed model "becomes" a period-2 cycle of the perturbed one. This is very close to what asserted by Proposition 1. However, the period-2 cycle arising in (5) does not consist, in general, of the two optimal production levels $q_{1}^{*}$ and $q_{2}^{*}$ of the perturbed optimization problem. To show this, it is sufficient to consider a very simple setting, in which the unperturbed demand function is linear. In the next example we implicitly assume suitable restrictions on the parameters so that $q^{*}, q_{i}^{*}$ and $\hat{q}_{i}$ are well-defined for any $\gamma$ and are strictly positive. When the inverse demand function is $p_{0}(q)=a q+b$ (where indeed $a<0$ and $b>0$ ), the unique equilibrium is

$$
q^{*}=-\frac{b-c}{2 a}
$$

\footnotetext{
${ }^{1}$ We stress that we (possibly) exclude the unique value $\gamma=-2 / \pi_{0}^{\prime \prime}\left(q^{*}\right)$ in order to be able to apply the implicit value theorem in its simplest formulation. With an additional assumption and a more technical proof, it is possible to encompass $\gamma=-2 / \pi_{0}^{\prime \prime}\left(q^{*}\right)$, too. Since it is just one value and then has a negligible relevance for our results, we avoided to consider it.
} 
while perturbed profit functions attain their maxima respectively at

$$
q_{1}^{*}(\varepsilon)=-\frac{b-c+h_{1}(\varepsilon)}{2 a}, \quad q_{2}^{*}(\varepsilon)=-\frac{b-c+h_{2}(\varepsilon)}{2 a} .
$$

Conversely, the unique period-2 cycle of the perturbed model (which can be easily obtained considering the steady state of System (6)) is given by

$$
\left\{\begin{array}{l}
\hat{q}_{1}^{\gamma}(\varepsilon)=\frac{2(c-b)-h_{1}(\varepsilon)-h_{2}(\varepsilon)+2 a \gamma\left(c-b-h_{2}(\varepsilon)\right)}{4 a(\gamma a+1)}, \\
\hat{q}_{2}^{\gamma}(\varepsilon)=\frac{2(c-b)-h_{1}(\varepsilon)-h_{2}(\varepsilon)+2 a \gamma\left(c-b-h_{1}(\varepsilon)\right)}{4 a(\gamma a+1)},
\end{array}\right.
$$

from which it is evident that $\hat{q}_{i}^{\gamma}(\varepsilon) \neq q_{i}^{*}(\varepsilon)$. Moreover, it's worth noticing that both $\hat{q}_{i}^{\gamma}(\varepsilon)$ directly depend on the agent reaction speed $\gamma$. This means that, differently from the classical unperturbed model in which the simple gradient mechanism is consistent with the static problem independently of $\gamma$ (namely the unique equilibrium is also a steady state of the model for any $\gamma$ ), in the perturbed model (5), in general, 2-cycles consist of values that do not correspond to optimal production choices of the two perturbed profit functions. Moreover, quite counterintuitively, the behavior of $\hat{q}_{1}^{\gamma}(\varepsilon)$ and $\hat{q}_{2}^{\gamma}(\varepsilon)$ can be completely different from that of $q_{1}^{*}(\varepsilon)$ and $q_{2}^{*}(\varepsilon)$, which, in agreement with Proposition 1, are respectively increasing and decreasing with $\varepsilon$. In fact, $\hat{q}_{1}^{\gamma}(\varepsilon)$ and $\hat{q}_{2}^{\gamma}(\varepsilon)$, depending on $\gamma$, can be both increasing and decreasing with respect to $\varepsilon$, as shown in Figure 1, from which we can see that the size of the oscillation can be both dampened and strengthened by the effect of $\gamma$.

Furthermore, if we consider a nonlinear demand function, the monotonicity of $\hat{q}_{1}^{\gamma}(\varepsilon)$ and $\hat{q}_{2}^{\gamma}(\varepsilon)$ as $\varepsilon$ increases can be lost, too. For example, we focus on a cubic inverse demand function

$$
p_{0}(q)=A-B q+D q^{2}-E q,
$$

similar to that proposed by Puu in [10], in which we choose coefficients so that the resulting marginal profit function can be written $\mathrm{as}^{2}$

$$
\pi_{0}^{\prime}(q)=a-2 b(q-k)+3 d(q-k)^{2}-4 e(q-k)^{3}-c .
$$

In doing so, parameter $k$ allows us to easily shift the marginal profit function (on which all results depend). Since the effect of the perturbation size on the resulting dynamics are unaffected by horizontal shifts of (9), this allows us to better highlight that the same effects can be obtained even when the perturbation size is small with respect to quantities. We remark that hereinafter all the parameters involved in (9) are chosen so that both assumptions (3) are satisfied, in order to have a unique optimal production level for each perturbed

\footnotetext{
${ }^{2}$ A straightforward computation shows that, in order to obtain (9) from $p_{0}$, we must impose $E=e, D=d+4 e k, B=-\left(-6 e k^{2}-3 d k-b\right)$ and $A=4 e k^{3}+3 d k^{2}+2 b k+a$.
} 

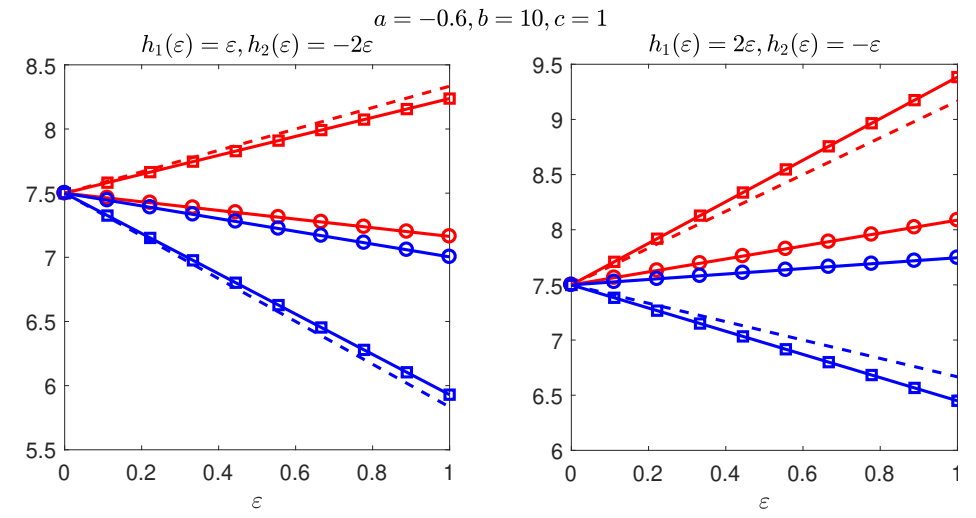

Figure 1: Left plot: asymmetric perturbation with $h_{1}(\varepsilon)=\varepsilon$ and $h_{2}(\varepsilon)=-2 \varepsilon$. Red and blue dashed lines respectively represent the evolution of $q_{1}^{*}(\varepsilon)$ and $q_{2}^{*}(\varepsilon)$. Red and blue solid lines respectively represent the evolution of $\hat{q}_{1}^{\gamma}(\varepsilon)$ and $\hat{q}_{2}^{\gamma}(\varepsilon)$ for $\gamma=0.1$ (circles) and $\gamma=0.8$ (squares). Right plot: asymmetric perturbation with $h_{1}(\varepsilon)=2 \varepsilon$ and $h_{2}(\varepsilon)=-\varepsilon$. Red and blue dashed lines respectively represent the evolution of $q_{1}^{*}(\varepsilon)$ and $q_{2}^{*}(\varepsilon)$. Red and blue solid lines respectively represent the evolution of $\hat{q}_{1}^{\gamma}(\varepsilon)$ and $\hat{q}_{2}^{\gamma}(\varepsilon)$ for $\gamma=0.2$ (circles) and $\gamma=0.9$ (squares).

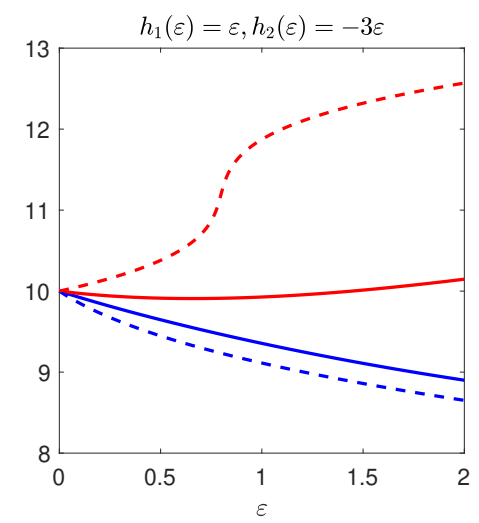

Figure 2: Evolution of $q_{1}^{*}(\varepsilon)$ and $q_{2}^{*}(\varepsilon)$ (red and blue dashed lines, respectively) and $\hat{q}_{1}^{\gamma}(\varepsilon)$ and $\hat{q}_{2}^{\gamma}(\varepsilon)$ for $\gamma=0.2$. (red and blue solid lines, respectively) when $p_{0}$ is a cubic polynomial affected by an asymmetric perturbation with $h_{1}(\varepsilon)=\varepsilon$ and $h_{2}(\varepsilon)=-3 \varepsilon$.

demand function. If we set $a=k=10, b=0.9, d=0.475$ and $e=0.1$, marginal cost $c=a, h_{1}(\varepsilon)=\varepsilon$ and $h_{2}(\varepsilon)=-3 \varepsilon$, we have that $\hat{q}_{1}^{\gamma}(\varepsilon)$ is decreasing when $\varepsilon \lesssim 0.65$ while it is increasing when $\varepsilon \gtrsim 0.65$, as shown in Figure 2 .

This means that in the perturbed model, without any precise knowledge of the underlying periodicity of the perturbation, the production decisions toward which trajectories converge are strongly influenced by the agent's behavior, can substantially vary for different values of $\gamma$ and, in general, do not correspond to the profit maximizing production decisions. 
In Proposition 2 we investigated the effect of perturbation $\varepsilon$ for a fixed reaction speed $\gamma$ of the agent. In the next Proposition we study the opposite situation, in which the economic setting, represented by the perturbation size $\varepsilon$, is fixed, and we vary the agent's behavior, in order to see how $\gamma$ affects period-2 cycles of the dynamical model (5). We may ask if, similarly to what happens when $\varepsilon_{i} \rightarrow 0^{+}$, as $\gamma \rightarrow 0^{+}$period-2 cycle $\left\{\hat{q}_{1}^{\varepsilon}(\gamma), \hat{q}_{2}^{\varepsilon}(\gamma)\right\}$ of (5) "converges" to the equilibrium of the unperturbed problem, namely if $\hat{q}_{i}^{\varepsilon}(\gamma) \rightarrow q^{*}$ for $\gamma \rightarrow 0^{+}$. However, such question is actually doomed in advance. In fact, for any given couple of "shifted" inverse demand functions $p_{1}$ and $p_{2}$ there are infinitely many "unperturbed" inverse demand functions $p_{0}(q)$, obtained choosing suitable perturbation values $h_{1}(\varepsilon)$ and $h_{2}(\varepsilon)$. Then, we can wonder which is the unperturbed problem to which 2-cycles of the non-autonomous equation (5) are, in some sense, related. We have the following Proposition, in which $\varepsilon_{M}$ is the value provided by Proposition 1.

Proposition 3. Let $\pi_{0}^{\prime}: \mathbb{R} \rightarrow \mathbb{R}$ be a differentiable function satisfying (4) and consider function

$$
\bar{\pi}^{\prime}(q, \varepsilon)=\pi_{0}^{\prime}(q)+\frac{h_{1}(\varepsilon)+h_{2}(\varepsilon)}{2},
$$

namely the average of the perturbed functions $\pi_{0}^{\prime}+h_{i}(\varepsilon)$. Let $\bar{q}^{*}(\varepsilon)$ be the unique zero of $\bar{\pi}^{\prime}(q)$. Then, for each $\varepsilon \in\left(0, \varepsilon_{M}\right)$, there are $\tilde{\gamma}(\varepsilon)>0$ and two differentiable functions $\hat{q}_{i}^{\varepsilon}: I_{\varepsilon}=(0, \tilde{\gamma}(\varepsilon)) \rightarrow \mathbb{R}, i=1,2$ such that

(1) $\left\{\hat{q}_{1}^{\varepsilon}(\gamma), \hat{q}_{2}^{\varepsilon}(\gamma)\right\}$ is a period-2 cycle of System (5) for any $\gamma \in I_{\varepsilon}$;

(2) $\lim _{\gamma \rightarrow 0^{+}} \hat{q}_{i}^{\varepsilon}(\gamma)=\bar{q}^{*}(\varepsilon)$;

(3) $\lim _{\gamma \rightarrow 0^{+}} \frac{d \hat{q}_{1}^{\varepsilon}}{d \gamma}=\frac{h_{1}(\varepsilon)-h_{2}(\varepsilon)}{4}, \lim _{\gamma \rightarrow 0^{+}} \frac{d \hat{q}_{2}^{\varepsilon}}{d \gamma}=-\frac{h_{1}(\varepsilon)-h_{2}(\varepsilon)}{4}$;

(4) $\hat{q}_{1}^{\varepsilon}(\gamma)$ is strictly increasing and $\hat{q}_{2}^{\varepsilon}(\gamma)$ is strictly decreasing.

We again have that a period-2 cycle of (5) arises from the unique solution of an optimization problem, which, however, involves the average of the two perturbed inverse demand functions. Thanks to the continuity of $\hat{q}_{i}^{\varepsilon}(\gamma)$, if the agents' reactivity is close to zero, the 2 -cycle has a very small amplitude around the optimal production choice of the average unperturbed problem. As $\gamma$ increases, we have that $\left|\hat{q}_{1}^{\varepsilon}(\gamma)-\hat{q}_{2}^{\varepsilon}(\gamma)\right|$ increases, too, so the oscillation extent increases as the agent becomes more reactive.

Nevertheless, we can compare the oscillation size $\left|\hat{q}_{1}^{\varepsilon}(\gamma)-\hat{q}_{2}^{\varepsilon}(\gamma)\right|$ of the period2 cycle of model (5) with the distance between the two optimal production levels $\left|\bar{q}_{1}^{*}(\varepsilon)-\bar{q}_{2}^{*}(\varepsilon)\right|$. The effect of $\gamma$ can be ambiguous. In fact, $\left|\hat{q}_{1}^{\varepsilon}(\gamma)-\hat{q}_{2}^{\varepsilon}(\gamma)\right|$ can be both smaller (for suitably small values of $\gamma$ ) and, possibly, larger (for suitably large values of $\gamma$ ) than $\left|q_{1}^{*}(\varepsilon)-q_{2}^{*}(\varepsilon)\right|$. Since $\hat{q}_{i}^{\varepsilon}(\gamma)$ are continuous monotonic functions, we can ask if there is a value $\gamma^{*}$ for which we simultaneously have $\hat{q}_{1}^{\varepsilon}(\gamma)=\bar{q}_{1}^{*}(\varepsilon)$ and $\hat{q}_{2}^{\varepsilon}(\gamma)=\bar{q}_{2}^{*}(\varepsilon)$, namely a particular agent's behavior allowing to alternate between the profit maximizing output levels. Imposing that 
$\left\{q_{1}^{*}(\varepsilon), q_{2}^{*}(\varepsilon)\right\}$ is a period-2 cycle of $(5)$, i.e.

$$
\left\{\begin{array}{l}
q_{1}^{*}(\varepsilon)=q_{2}^{*}(\varepsilon)+\gamma\left(\pi_{0}^{\prime}\left(q_{2}^{*}(\varepsilon)\right)+h_{1}(\varepsilon)\right) \\
q_{2}^{*}(\varepsilon)=q_{1}^{*}(\varepsilon)+\gamma\left(\pi_{0}^{\prime}\left(q_{1}^{*}(\varepsilon)\right)+h_{2}(\varepsilon)\right)
\end{array}\right.
$$

we find

$$
\gamma^{*}=\frac{q_{1}^{*}(\varepsilon)-q_{2}^{*}(\varepsilon)}{h_{1}(\varepsilon)-h_{2}(\varepsilon)},
$$

so, in principle, there always exists a particular reaction speed for which the period-2 cycle of the perturbed model oscillates between the two profit maximizing production choices. We recall that in the classical unperturbed monopoly model the equilibrium $q^{*}$ is a steady state of the model independently of $\gamma$ and that it is stable provided that $\gamma \in(0,1] \cap(0, \tilde{\gamma})$, for some stability threshold $\tilde{\gamma}>0$. This means that a suitably cautious behavior of the agent always allows trajectories to converge toward the equilibrium $q^{*}$ (at least those starting in a neighborhood of $q^{*}$ ). Conversely, in the perturbed model it is no more true, and even if the period-2 cycle is stable, it is increasingly different from $\left\{q_{1}^{*}, q_{2}^{*}\right\}$ as $\gamma$ is either increasingly smaller or larger than $\gamma^{*}$. From Proposition 3, we can conclude that the economic agent, just observing and taking into account the profitability change signal, even if does not shape his/her decision mechanism on a precise knowledge of the underlying time variability of the demand function, can learn the qualitative cyclicity of the demand function, and react to it in a qualitatively correct way. In fact, the right ordering between production choices when the market size is either large or small is preserved (point 4 of Proposition 3, Figures 1,2 and 3). However, he/she is in general not able to learn the correct optimal production levels. As a consequence, both a too cautious and a too reactive behavior induce reduced asymptotic profits. Finally we remark that $\gamma^{*}$ may not lie inside interval $(0,1]$ so we may have situations in which the optimal productions levels $q_{i}^{*}$ are never points of a 2-cycle of (5), unless considering overreaction. As we will show in Section 4, the deviation between optimal profits and those actually realized adopting the present mechanism is in general negligible, especially when the period- 2 cycle is stable.

To exemplify the previous results, we make again reference to the case of a linear demand function, for which $q_{i}^{*}$ are indeed constant with respect to $\gamma$ (see (7)) and the expressions of $\hat{q}_{i}^{\varepsilon}(\gamma)$ are those given by (8). A simple computation shows that $d \hat{q}_{1}^{\varepsilon} / d \gamma=\left(h_{1}(\varepsilon)-h_{2}(\varepsilon)\right) /\left(4(a \gamma+1)^{2}>0\right)$ and $d \hat{q}_{2}^{\varepsilon} / d \gamma=-d \hat{q}_{1}^{\varepsilon} / d \gamma<$ 0 , in agreement with the results of Proposition 3. This also implies that the size of the oscillation $\left|\hat{q}_{1}^{\varepsilon}(\gamma)-\hat{q}_{2}^{\varepsilon}(\gamma)\right|$ increases on increasing $\gamma$. Moreover, if we directly solve $\hat{q}_{i}^{\varepsilon}(\gamma)=q_{i}^{*}$ we find $\gamma^{*}=-1 /(2 a)$ (the same result is obtained by directly applying (10)). This means that if $a \leq-0.5$ we have $\gamma^{*} \in(0,1]$, while for $a>-0.5$ the optimal productions levels $q_{i}^{*}$ are never points of a 2-cycle of the linear model. The situation is illustrated in Figure 3. We notice that the perturbation is asymmetric and for $\gamma \rightarrow 0^{+}$both $\hat{q}_{i}^{\varepsilon}(\gamma)$ converge to the average of the perturbed equilibria.

We remark that in both Propositions 2 and 3, the period-2 cycle arising from $q^{*}$ or $\bar{q}^{*}(\varepsilon)$ may not exist for every $\varepsilon$ (in Proposition 2) or for every $\gamma \in(0,1]$ 

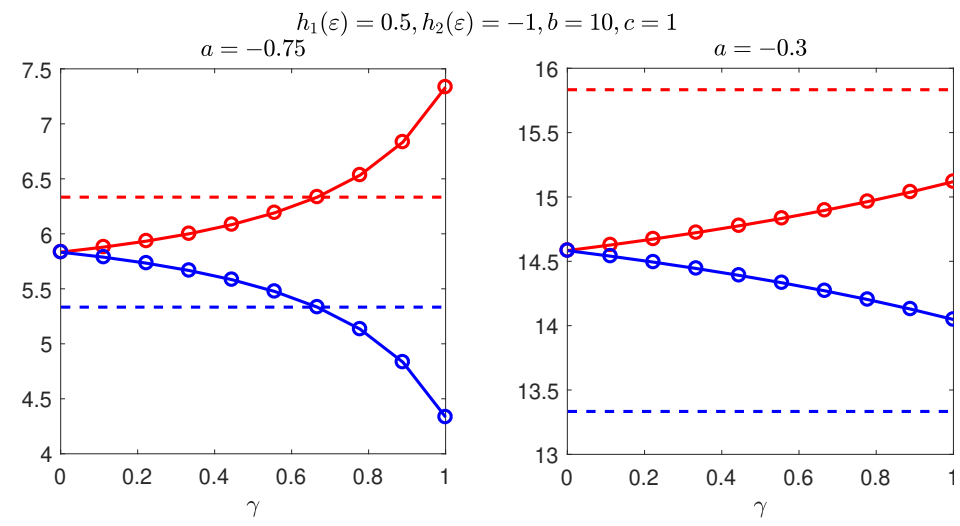

Figure 3: Red and blue dashed lines respectively represent $q_{1}^{*}$ and $q_{2}^{*}$, which are constant with respect to $\gamma$. Red and blue solid lines respectively represent the evolution of $\hat{q}_{1}^{\varepsilon}(\gamma)$ and $\hat{q}_{2}^{\varepsilon}(\gamma)$ for $h_{1}(\varepsilon)=0.5$ and $h_{2}(\varepsilon)=-1$. Both $\hat{q}_{i}^{\varepsilon}(\gamma)$ are increasing, but if $a \leq-0.5$ there exists a $\gamma^{*}$ at which $\hat{q}_{i}^{\varepsilon}\left(\gamma^{*}\right)=q_{i}^{*}$ (left plot, if $a=-0.6$ we find $\gamma^{*}=2 / 3$ ) while if $a>-0.5$ it is not possible (right plot, $a=-0.3$ ).

(in Proposition 3), even if the corresponding couple of points $q_{1}^{*}$ and $q_{2}^{*}$ exists for any $\varepsilon$. This is essentially an effect of the agent's reaction, which then does not only affect the actual period-2 cycle of the perturbed model, but even its existence. Such aspect will be further investigated in Section 4.

We also stress that in Propositions 2 and 3 we showed that there exists a period-2 cycle of the perturbed model which corresponds to the optimal output level of an unperturbed problem (which can be the original unperturbed model or the average unperturbed one). As we will show in Section 4, it may not be the unique period-2 cycle of model (5).

In Proposition 3 we showed that, differently from the classical framework, the steady state $\left(\hat{q}_{1}^{\varepsilon}(\gamma), \hat{q}_{2}^{\varepsilon}(\gamma)\right)$ of $(6)$ is affected by $\gamma$ and trajectories may not converge toward the profit maximizing output levels. However, convergence can be not possible also in the classical framework, in which $\gamma$ has in general a destabilizing effect on the steady state. So far, we just studied the existence of period-2 cycles, which however, can be unstable. We study the local asymptotic stability of a generic period-2 cycle $\left\{\hat{q}_{1}, \hat{q}_{2}\right\}$ in the next Proposition.

Proposition 4. Let $\left\{\hat{q}_{1}, \hat{q}_{2}\right\}$ be a period-2 cycle of the non-autonomous equation (5). Then it is locally asymptotically stable provided that

$$
\left|\left(1+\gamma \pi_{0}^{\prime \prime}\left(\hat{q}_{1}\right)\right)\left(1+\gamma \pi_{0}^{\prime \prime}\left(\hat{q}_{2}\right)\right)\right|<1 .
$$

Stability condition (11) directly depends on $\gamma$, being it the coefficient of $\pi_{0}^{\prime \prime}$. Moreover, since $\left\{\hat{q}_{1}, \hat{q}_{2}\right\}$ depends on both the agent's reactivity and the perturbation size, $\gamma$ and $\varepsilon$ both indirectly affects stability through their influence on the resulting period- 2 cycle. One of the main consequences is that $\gamma$ can have an ambiguous effect on stability. To this end, an essential role is played by the 
concavity of the marginal profit function. If we considered a linear demand function, we would simply have that $(11)$ reduces to $(1+2 a \gamma)^{2}<1$. Stability is then unaffected by the perturbation, while $\gamma$ only has the usual destabilizing role. Conversely, in the nonlinear case, we have a more articulated situation. The possible effects of $\gamma$ in a simple case are investigated in the next Corollary, in which, for easy, we consider a symmetric perturbation so that $\bar{\pi}=\pi_{0}$.

Corollary 1. Let us consider a fixed value of $\varepsilon$. Then the following scenarios are possible:

(1a) $\left\{\hat{q}_{1}, \hat{q}_{2}\right\}$ is stable for any $\gamma \in(0,1]$;

(1b) there is $\gamma_{p d} \in(0,1)$ so that $\left\{\hat{q}_{1}, \hat{q}_{2}\right\}$ is locally asymptotically stable for $\gamma \in\left(0, \gamma_{p d}\right)$ and unstable for $\gamma \in\left(\gamma_{p d}, 1\right]$;

(1c) there are $0<\gamma_{p d}<\gamma_{p h}<1$ so that $\left\{\hat{q}_{1}, \hat{q}_{2}\right\}$ is locally asymptotically stable for $\gamma \in\left(0, \gamma_{p d}\right) \cup\left(\gamma_{p h}, 1\right]$ and unstable for $\gamma \in\left(\gamma_{p d}, \gamma_{p h}\right)$.

The previous Corollary shows that, unlike the classical unperturbed situation, the effect of introducing a perturbation allows parameter $\gamma$ to have a stabilizing effect, too. In particular, in case (2c), a bubbling phenomenon occurs, with a return to stability when the agents reaction speed increases.

The actual occurrence of each scenario will be shown through simulations in Section 4 , in which we will use a cubic inverse demand function. We underline that the possibility for a demand function to change its concavity (and as a possible consequence, for the marginal profit function, too) is not just a mere mathematical concern, but it can concretely represent a consequence of the consumers' behavior or heterogeneity, as noticed by Robinson in [3] and reported by Puu in [10].

We remark that from Proposition 4 , it is evident that the local asymptotic stability of the period-2 cycle $\left\{\hat{q}_{1}, \hat{q}_{2}\right\}$ is completely independent of the stability of equilibrium $q^{*}$ in the dynamical unperturbed model. In principle, this means that introducing the perturbation may have both a stabilizing and a destabilizing effect. We will investigate this aspect, together the possible scenarios as the perturbation size increases, in Section 4.

\section{Numerical experiments}

In all the simulations reported in this section we consider a (unperturbed) cubic inverse demand function which provides the cubic profit function (9). As shown in [10] if $a, b$ and $e$ are positive and if $d^{2}-3 b e<0$, the cubic function $p_{0}$ is decreasing. In what follows, unless otherwise specified, we set $a=c=100$ and $k=10$. Remaining parameters and perturbations are chosen so that all the economically significant quantities and equilibria, in both unperturbed and perturbed models, are positive as $\gamma$ and $\varepsilon$ increase. Finally, unless otherwise specified, we computed all the bifurcation diagrams "following" the attractor, namely if the bifurcation diagram is computed for a sequence $\left\{a_{i}\right\}$ of parameters, 


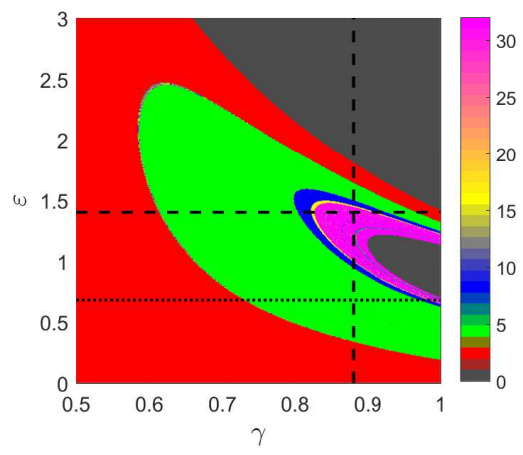

(A)

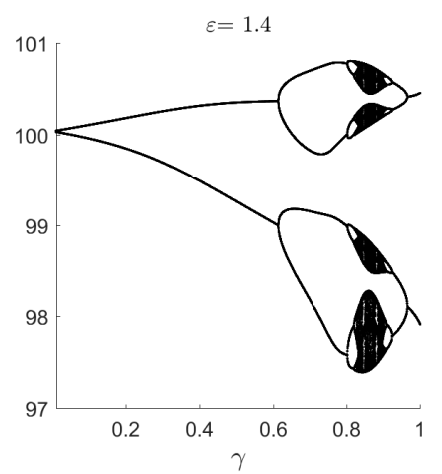

(C)

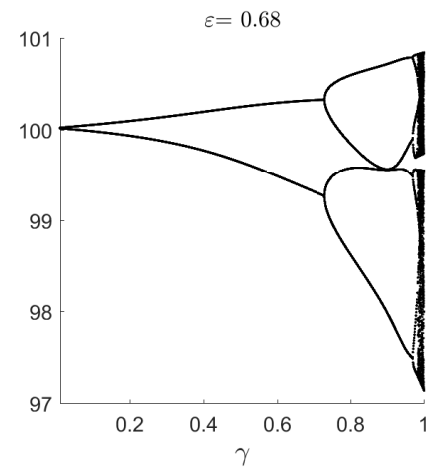

(B)

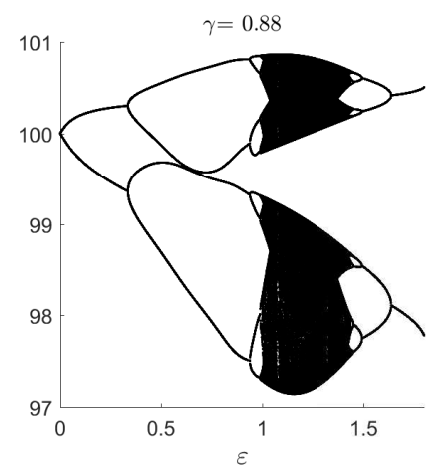

(D)

Figure 4: (A): two-dimensional bifurcation diagram with respect to $\gamma$ and $\varepsilon$. Color red is used for period-2 cycles. Color dark gray represents parameters that provide diverging trajectories. Remaining colors are used for attractors consisting of more than a single point. (B,C): bifurcation diagrams, as $\gamma$ increases, when $\varepsilon=0.68$ (plot (B), corresponding to the horizontal dotted line in Figure (A)) and $\varepsilon=1.4$ (plot (C), corresponding to the horizontal dashed line in Figure (A)). (D): bifurcation diagram, as $\varepsilon$ increases, for $\gamma=0.88$ (corresponding to the vertical dashed line in Figure (A)).

the initial datum for the simulation corresponding to $a_{i+1}$ is chosen suitably close to a point of the attractor toward which trajectories obtained with parameter $a_{i}$ converged. The goal of this Section is to illustrate the results of Section 3 and to deepen their investigation with the help of numerical simulations. We are mainly interested in exhibiting the main theoretical peculiarities of the periodically perturbed setting with respect to the classical one. We start sketching the interpretation and significance of such differences, whose economic relevance will become evident in Section 5 in the comparison with the economic literature.

In the first two families of simulations we investigate the dynamical behavior of the perturbed model with respect to agent's reaction speed $\gamma$ and the size of perturbation $\varepsilon$. In Figure 4 (A) we report a two-dimensional bifurcation 
diagram obtained setting $b=1, d=-0.54$ and $e=0.11$. We consider the asymmetric perturbation defined by $h_{1}(\varepsilon)=\varepsilon$ and $h_{2}(\varepsilon)=-0.9 \varepsilon$. In this case, it is easy to see that the unique equilibrium $q^{*}=10$ of the unperturbed model is unconditionally stable for any $\gamma \in(0,1)$. It is evident that for suitably small values of $\gamma$ and $\varepsilon$, the period-2 cycle is stable (red region in Figure 4 (A)). Keeping fixed $\varepsilon$, we have that $\left\{\hat{q}_{1}^{\varepsilon}(\gamma), \hat{q}_{2}^{\varepsilon}(\gamma)\right\}$ is unconditionally stable for any $\gamma \in(0,1]$ provided that $\varepsilon \lesssim 0.19$ (scenario (1a), Corollary 1). For intermediate values of the perturbation size $(0.19 \lesssim \varepsilon \lesssim 1.31)$, period-2 cycle $\left\{\hat{q}_{1}^{\varepsilon}(\gamma), \hat{q}_{2}^{\varepsilon}(\gamma)\right\}$ becomes unstable through a flip bifurcation, as shown in Figure 4 (A) when horizontal lines cross at some $\tilde{\gamma}$ the boundary of the green region, in which trajectories converge toward period-4 cycles (scenario (1b), Corollary 1). Such cycles can then remain stable for any $\gamma>\tilde{\gamma}$ or they can undergo a cascade of period-doublings, as for example reported in Figure 4 (B), from which we can notice the emergence of chaotic dynamics for large reaction speeds. However, as $\varepsilon$ approaches 1.31 , we can notice from Figure 4 (A) a qualitative simplification of the dynamics, in particular for $\gamma$ sufficiently close to 1 . When $1.31 \lesssim \varepsilon \lesssim 2.48$ we have a bubbling in the bifurcation diagram. As shown in Figure $4(\mathrm{C})$, the initial flip bifurcation undergoes a cascade of period-doublings leading to chaos, which is then followed by a cascade of period-halvings so that, for sufficiently large values of $\gamma,\left\{\hat{q}_{1}^{\varepsilon}(\gamma), \hat{q}_{2}^{\varepsilon}(\gamma)\right\}$ is again stable (scenario (1c), Corollary 1). As $\varepsilon$ increases, the resulting dynamics further simplify, and the bubbling possibly consists of a single period-doubling followed by a single period-halving. Finally, for $\varepsilon \gtrsim 2.48$, the period-2 cycle is again unconditionally stable with respect to $\gamma$, as long as it exists. Since the equilibrium in the unperturbed model is stable, all the above mentioned phenomena are introduced by the perturbation, and then affected by the reaction speed. Small perturbations have a destabilizing effect, introducing possible unstable, periodic or chaotic dynamics which are not present in the unperturbed model, while larger perturbations may have the counterintuitive effect of improving and even recovering stability. We remark that as $\gamma$ approaches $0^{+}$(as in the leftmost parts of the bifurcation diagrams reported in Figures 4 (B) and (C)), we have that the size of the oscillation becomes increasingly small, but the attractor still consists of two distinct points for any $\gamma>0$, in agreement with Proposition 3.

The bifurcation diagram reported in Figure $4(\mathrm{C})$ deserves some comments. In the classical time-constant setting, the output decisions coincide with the steady state if the marginal profit is null. Moreover, in a neighborhood of such steady state the agent's reactivity can be either "small" (relatively to the profitability change signal) or "large".

As in the classical framework, in a time-varying setting, the overall reactivity encompassed in the decision mechanism is the result of the joint effect of the endogenous agent's reactivity to profit variations and of the strength of the profitability signal. The main difference is that with a period-2 demand function we can actually distinguish two alternating market phases, each one identified by its own demand function. Since we assumed that demand functions vertically shift, this actually corresponds to considering a market which assumes two different, alternating sizes, which we can respectively identify with the "peak" 
and the "off-peak" phases of the market. Indeed, when passing from a peak to an off-peak (respectively from an off-peak to a peak) phase, the profitability signal will in general suggest to decrease (respectively increase) the output level. However, the variation of the production decisions between two consecutive either peak or off-peak phases (i.e. from $t$ to $t+2$ ) is decided on the basis of the joint effect of two consecutive profitability change signals ${ }^{3}$. If we consider the simple but general situation of a symmetric perturbation $\left(h_{1}=h_{2}\right)$, looking at the second equation of (6), we have that the overall signal between two consecutive off-peak (respectively peak) phases is exactly given by the sum of the two consecutive marginal profits, namely $\pi_{0}^{\prime}\left(q_{2, t}\right)+\pi_{0}^{\prime}\left(q_{1, t+1}\right)$ (respectively $\left.\pi_{0}^{\prime}\left(q_{1, t}\right)+\pi_{0}^{\prime}\left(q_{2, t+1}\right)\right)$. In general, the steady state output level of a phase is then reached not when marginal profits are both null, but when they are equal and opposite. In a neighborhood of the steady state we have in general that the profitability variation signals of a phase can be different from that of the other one. As a consequence, the agent's reactivity can be suitably "small" at a market occurrence and "large" at the other one.

To understand the possible evolution of the production decisions, we must take into account the previous considerations. To fix ideas, we assume that marginal profits are relatively small in the off-peak phase and relatively large in the peak one. We set at a peak phase, assuming that the output decision is suitably close to and smaller than the steady state of the peak phase and we focus on what happens between two consecutive peak phases (the line of reasoning can be easily adapted to study two consecutive off-peak phases). The adjustment of the production decision for the next off-peak phase is negative but relatively small and it is followed by a larger positive adjustment, since we assumed that marginal profits are larger near the steady state of the peak phase. The overall profitability variation signal between two consecutive peak phases is then positive.

If the agent's reactivity is sufficiently small, the adjustment between two consecutive peak phases is small. The new output decision for the peak phase is again smaller than that at the peak steady state and, going on, output decisions of the peak phase are progressively adapted toward it. The resulting adjustment process can be slow, but its direction is constant: production decisions carry on increasing, following a monotonic trajectory toward the peak steady state.

If the agent's reactivity is larger, convergence is potentially faster. However, this can lead to an overestimation of the production level. If the agent's reactivity is not too large, the new production level is closer to that at the peak steady state, but in this case it is larger than it. We notice that since the agent's reaction is still relatively small with respect to the size of the profitability signal, the production level only slightly decreases, and the resulting output decision for the off-peak phase is overestimated, too. Both overestimations lead the overall marginal profits to become negative for the next couple of phases.

${ }^{3}$ From then mathematical point of view, this means that the dynamical behavior is determined by maps $f_{1}\left(f_{2}(q)\right)$ and $f_{2}\left(f_{1}(q)\right)$. 
This will make the corresponding output level to be underestimated, with a reverse adjustment with respect to the past one. In each phase, production levels then alternate between decreasingly too large and too small output levels, which converge following non-monotonic trajectories.

If the agent's reactivity further increases, the reaction to the overall profitability signal may drift the next output decision apart from the possible peak steady state. If we considered a market consisting of the only off-peak phase with an agent characterized by such reactivity, the production decisions would converge, but slowly, since the agent's reactivity is still small with respect to the marginal profit signal. The deviation due to the stronger profitability variation signal coming from the peak phase together with a relatively large reaction speed is then that dominating and the next peak output level moves away from the peak steady state. In this situation, production decisions of each phase can follow complex trajectories, arising endogenously, which can be indistinguishable from random erratic exogenous movements (for further discussions about chaos and random shocks we refer to [?]).

However, as the reaction speed of the agent further increases, we possibly have a simplification of the last scenario. Even if we again have an overestimation of the next peak production levels, the agent's reaction can be suitable to obtain a fast convergence of the production level in the off-peak phase, so that the production decision move back closer to the steady state. The effect of two opposite signals from the marginal profit estimation leads the production decisions into approaching again the steady state and the output trajectories again converge.

Finally, if the reaction of the agent becomes too large with respect to both peak and off-peak marginal profits, output trajectories of both phases can diverge.

The ambiguous behavior with respect to the perturbation size is confirmed if we look again at the two-dimensional bifurcation diagram of Figure 4 and we let $\varepsilon$ vary, keeping $\gamma$ fixed. For a small agent's reaction speed, period-2 cycle $\left\{\hat{q}_{1}^{\gamma}(\varepsilon), \hat{q}_{2}^{\gamma}(\varepsilon)\right\}$ is stable with respect to $\varepsilon$. For sufficiently large values of $\gamma$ we again have a bubbling in the bifurcation diagram, so that the period-2 cycle is unstable for intermediate perturbation sizes and stable for suitably either small or large values of $\varepsilon$, as shown in Figure 4 (D).

As already noticed, it is evident how a small perturbation can introduce instability. Since a large perturbation can have the opposite effect, we can ask whether the introduction of a perturbation in an unperturbed model with unstable dynamics can improve stability, too. This is investigated in the next family of simulations. In Figure 5 (A) we report the two-dimensional bifurcation diagram obtained for $b=2, d=0.73$ and $e=0.11$ and for the asymmetric perturbation defined by $h_{1}(\varepsilon)=\varepsilon$ and $h_{2}(\varepsilon)=-0.1 \varepsilon$. Before considering what happens as $\varepsilon$ varies, we briefly focus again on the effect of the reaction speed. In this case, the equilibrium $q^{*}=10$ of the unperturbed model is stable only for $\gamma<0.5$. As we can see, the introduction of a sufficiently large perturbation again has a stabilizing effect on the dynamics on varying $\gamma$. In the simulation reported in Figure 5 (B), we have that the period-2 cycle $\left\{\hat{q}_{1}^{\varepsilon}(\gamma), \hat{q}_{2}^{\varepsilon}(\gamma)\right\}$ can be 
stable even if the unperturbed model exhibits chaotic dynamics. We notice that it is evident that as $\gamma \rightarrow 0^{+}, \hat{q}_{i}^{\varepsilon}(\gamma)$ does not converge toward $q^{*}$, in agreement with Proposition 3. Moreover, looking at Figure 5 (A), when $\varepsilon \gtrsim 3.25$, we have unconditional stability with respect to $\gamma$. Setting $\gamma=0.66$, for which in the unperturbed model trajectories converge to a period- 4 cycle (red bifurcation diagram of Figure $5(\mathrm{~B})$ ), we have that increasing the perturbation size has the initial effect of increasing the complexity of the dynamics (Figure $5(\mathrm{C})$ ). This also means that, even when the perturbation size is small, a small change in the demand oscillation extent can induce significant changes in the qualitative behavior of the dynamics. However, when $\varepsilon$ is sufficiently large, a cascade of period-halvings occurs and $\left\{\hat{q}_{1}^{\gamma}(\varepsilon), \hat{q}_{2}^{\gamma}(\varepsilon)\right\}$ becomes stable again. Moreover, such stabilization can occur even if the unperturbed model is characterized by chaotic dynamics, as shown in Figure 5 (D).

The interpretation of the effect of the variation of the market size is simpler with respect to that with respect to the agent's reactivity. In this case, we have that as the demand function changes, the marginal profits at the (new) steady states of peak/off-peak market phases change, too. This means that the profitability variation signal can become either stronger or weaker (in each phase). Recalling what we said about the joint effect of such signal and of the agent's reactivity, this can induce either an increase or a simplification of the complexity of output trajectories.

Considering a cubic inverse demand function, it is evident that $q_{i}^{*}$ are welldefined for any perturbation size, at least from the mathematical viewpoint, as $q_{2}^{*}(\varepsilon)$ becomes negative for suitably large values of $\varepsilon$. However, recalling Proposition 2, period-2 cycle $\left\{q_{1}^{\gamma}(\varepsilon), q_{2}^{\gamma}(\varepsilon)\right\}$ may not exist for any perturbation size. In fact, if we look at the two-dimensional bifurcation diagrams of Figures 4 and 5, we can see that red regions, in which the period-2 cycle is stable, can share the boundary with dark gray regions, in which trajectories diverge. This is due to a couple of fold bifurcations in maps $f_{1}\left(f_{2}(q)\right)$ and $f_{2}\left(f_{1}(q)\right)$ where functions $f_{i}$ are defined in (5). For the same parameter set used for the bifurcation diagram reported in Figure $4(\mathrm{D})$, in Figure 6 (A) we report maps $f_{1}\left(f_{2}(q)\right)$ and $f_{2}\left(f_{1}(q)\right)$ for perturbation sizes immediately below and above the fold bifurcation threshold, together with a cobweb diagram (Figure 6 (B)) referred to maps $q_{2}+\gamma\left(\pi_{0}^{\prime}\left(q_{2}\right)+h_{1}(\varepsilon)\right)$ (in red) and $q_{1}+\gamma\left(\pi_{0}^{\prime}\left(q_{1}\right)+h_{2}(\varepsilon)\right.$ ) (in blue). To have a fold bifurcation we must have

$$
\left(1+\gamma \pi_{0}^{\prime \prime}\left(\hat{q}_{1}\right)\right)\left(1+\gamma \pi_{0}^{\prime \prime}\left(\hat{q}_{2}\right)\right)=1,
$$

together with some supplementary conditions (see [27], Theorem 1). Indeed, a sufficient condition for (12) is to simultaneously have $\pi_{0}^{\prime \prime}\left(\hat{q}_{1}\right)=\pi_{0}^{\prime \prime}\left(\hat{q}_{2}\right)=0$, which is, in general, not necessary. In Figure 6 (B) we considered a perturbation size very close to the threshold of the fold bifurcation, and we numerically find $\pi_{0}^{\prime \prime}\left(\hat{q}_{1}\right)=-1.56$ and $\pi_{0}^{\prime \prime}\left(\hat{q}_{2}\right)=-4.1$, for which the left hand side of (12) is equal to 0.988 . We stress that looking at Figure 6 (B), we can notice that System (6) has also other steady states, which are however locally asymptotically unstable. 


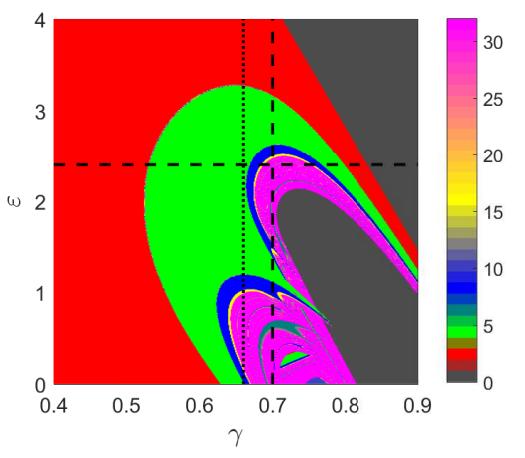

(A)

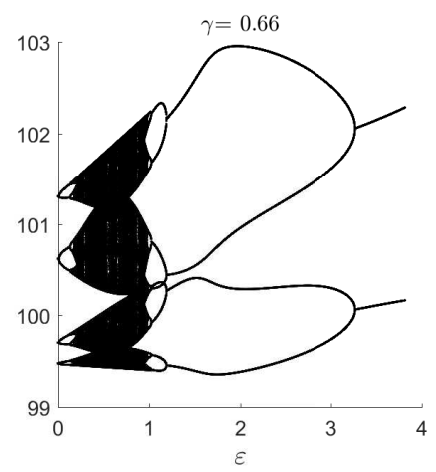

(C)

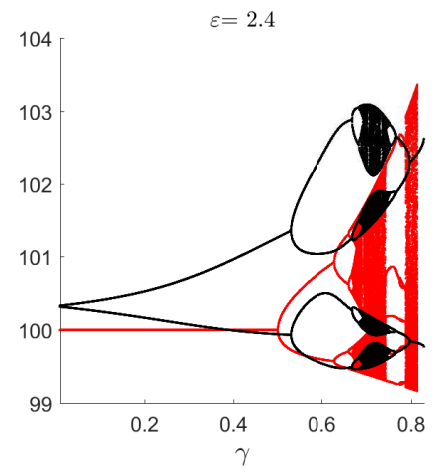

(B)

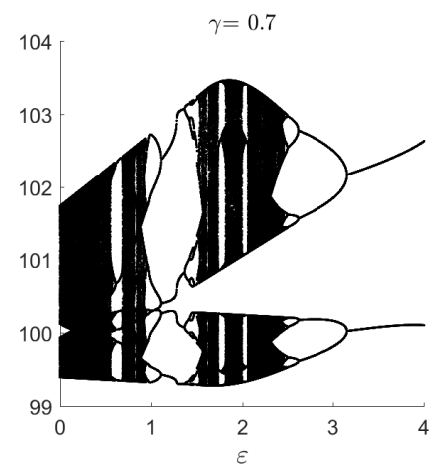

(D)

Figure 5: (A): two-dimensional bifurcation diagram with respect to $\gamma$ and $\varepsilon$. Color red is used for period- 2 cycles. Color dark gray represents parameters that provide divergence of trajectories. Remaining colors are used for attractors consisting of more than a single point. (B): bifurcation diagrams, as $\gamma$ increases, for the perturbed model (black) and the unperturbed one (red), for $\varepsilon=2.4$ (corresponding to the horizontal dashed line in Figure (A)). (C): bifurcation diagram, as $\varepsilon$ increases, when $\gamma=0.66$ (corresponding to the vertical dotted line in Figure (A)). (D): bifurcation diagram, as $\varepsilon$ increases, when $\gamma=0.7$ (corresponding to the vertical dashed line in Figure (A)).

Finally, we just want to quickly point out that the period-2 cycle arising from the equilibrium $q^{*}$ of the unperturbed model may not be the unique period-2 attractor of (5). In Figure 7 we show two bifurcation diagrams with respect to $\gamma$, obtained for the same parameter configuration used for the simulation reported in Figure 5, for a small perturbation size $\varepsilon=0.05$ and considering two different initial data $q_{2,0}=10.3$ and $q_{2,0}=12$. As we can see, for $\gamma<0.53$, both trajectories converge to the period-2 cycle arising from $q^{*}$, so the black and the red bifurcation diagrams coincide. When $\gamma \gtrsim 0.53$, the trajectories obtained considering $q_{2,0}=10.3$ still converge to the period- 2 cycle arising from $q^{*}$, while if we take $q_{2,0}=12$ trajectories converge to another stable period- 2 cy- 


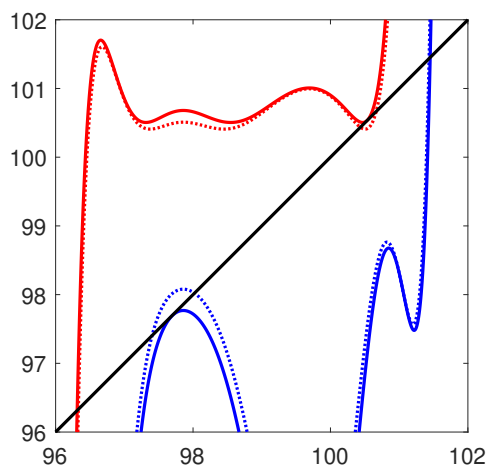

(A)

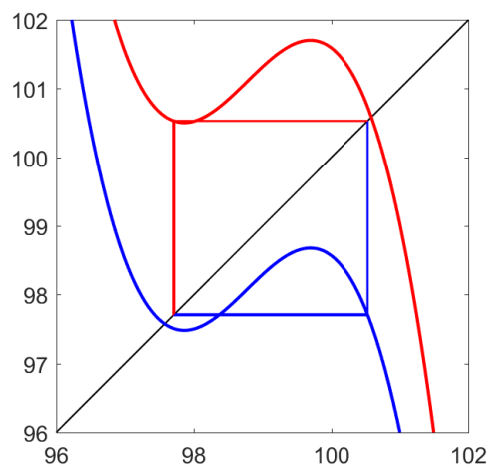

(B)

Figure 6: (A): maps $f_{1}\left(f_{2}(q)\right)$ and $f_{2}\left(f_{1}(q)\right)$ (red and blue color). Maps corresponding to $\varepsilon=$ 1.7 are represented using dotted lines, while those corresponding to $\varepsilon=1.808$ are represented using solid lines. (B): cobweb diagram for the non-autonomous equation $(5)$ when $\varepsilon \approx 1.808$. Red and blue colors respectively represent the maps defined by the right hand side of (5) when $t$ is either odd or even.

cle which coexists with the original stable period-2 cycle, as pointed out by the "jump" in the red bifurcation diagram. They both give then rise to a cascade of period-doublings leading, for different values of the reaction speed, to chaotic dynamics. The emergence of such coexistence corresponds to a couple of fold bifurcations, each one respectively affecting $g_{1}$ and $g_{2}$. Such phenomenon was already observed and studied in [25] for the periodically perturbed logistic equation. In that case, it was possible to cast an analytical glance at the interval of possible perturbation sizes for which coexistence arose, computing the resultant of the system (a fourth order polynomial) and approximating it by means of the Newton polygon procedure. In the present situation, a similar approach is actually impossible, since the resultant of (6) is a very high degree polynomial, so we limit to show the emergence of an analogous behavior, reporting in Figure 7 (B) the cobweb diagram for the two attractors when $\gamma=0.65$, together with the related basins of attractions. It's worth noticing that such coexistence is not induced by the particular demand function or by the agent's behavior, since we thoroughly checked through numerical simulations that when $\varepsilon=0$ no coexisting attractor with positive measure seems to emerge. It is then introduced by the perturbation itself, as in [25].

From the propositions of Section 3 and from the simulations reported in this Section, it is evident that a mechanism like (5) is (in general) not able to let the agent "learn" the profit maximizing productions levels. We recall that this is a consequence of the rationality assumption for the agent, who is not aware of the precise time variability of the demand function. We may wonder how much achieved profits are actually affected by such boundedly rational adjustment 


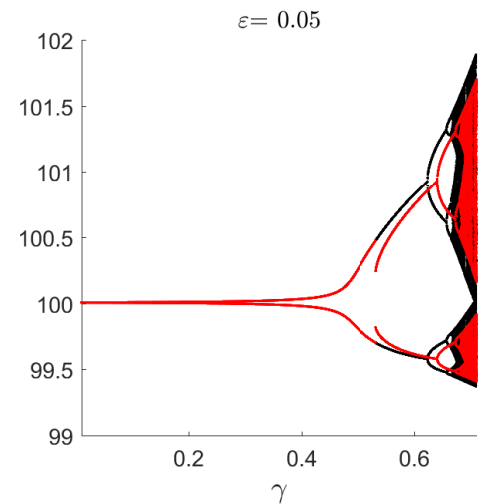

(A)

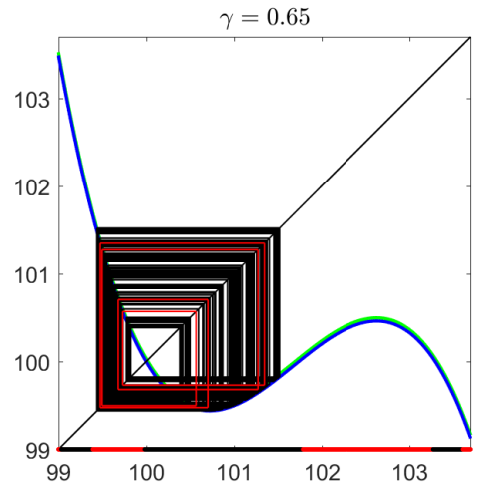

(B)

Figure 7: (A): bifurcation diagrams for two different initial data $\left(q_{2,0}=100.3\right.$ for the black diagram and $q_{2,0}=102$ for the red diagram), for the parameter configuration of the simulation reported in Figure 5 (A). (B): cobweb diagram for two different initial data, showing coexistence between a period- 8 cycle (red) and chaotic trajectories (black). Maps $f_{1}$ and $f_{2}$ are represented using green and blue color. Basins of attraction are reported at the bottom of the plot. Each initial datum is represented using the color of the attractor toward which the corresponding trajectory converges.

process. To investigate this we focus on a situation in which the perturbation is quite significant, considering $a=c=k=4, b=1.4, d=0.618$ and $e=-0.11$ and a symmetric perturbation $h_{1}(\varepsilon)=-h_{2}(\varepsilon)=\varepsilon$. In Figures $8(\mathrm{~A})$ and $(\mathrm{B})$ we reported the upper and the lower parts of the bifurcation diagram with respect to $\gamma$ obtained setting $\varepsilon=1$. It is evident that, both when $t$ is odd and even, the distance between the best profits (represented by the dashed line) and the achieved profits is mild, especially when dynamics converge toward the period-2 cycle.

To check the robustness of the previous result, we can estimate the relative distance between realized and achieved profits as

$$
D=\frac{\left|\hat{\pi}_{1}-\pi_{1}^{*}\right|}{2 \pi_{1}^{*}}+\frac{\left|\hat{\pi}_{2}-\pi_{2}^{*}\right|}{2 \pi_{2}^{*}}
$$

where $\hat{\pi}_{1}, \hat{\pi}_{2}$ and $\pi_{1}^{*}, \pi_{2}^{*}$ respectively represent the average realized profits and the best profits when $t$ is odd and when $t$ is even. In Figure $8(\mathrm{C})$ we report the two-dimensional bifurcation diagram while in Figure $8(\mathrm{C})$ we report index $100 \cdot D$, in which averages are computed over 200 time periods. As we can see, the distance is always reasonably small. We stress that, even if such results are obtained by considering a symmetric perturbation, we numerically checked that they are not significantly altered by (even strongly) asymmetric perturbations.

Finally, we notice that in a realistic situation in which a stochastic shock is over-imposed to the deterministic cyclicity of the demand, it can be very difficult 


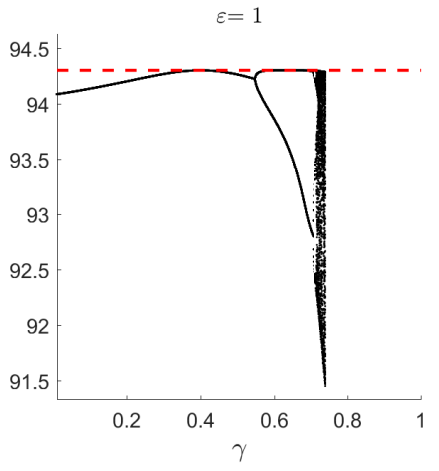

(A)

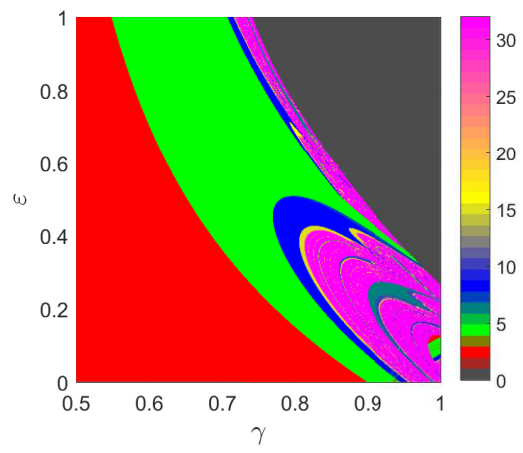

(C)

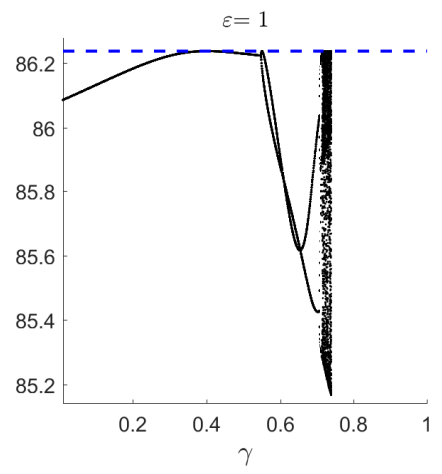

(B)

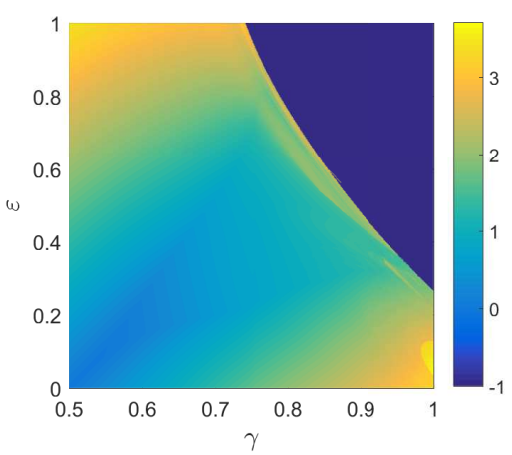

(D)

Figure 8: (A,B): bifurcation diagram of profits as $\gamma$ increases, when $\varepsilon=1$. In plot $(\mathrm{A})$ we report profits when $t$ is odd, in plot (B) we report profits when $t$ is even. Dashed lines represent optimal profits. (C): two-dimensional bifurcation diagram with respect to $\gamma$ and $\varepsilon$. Color red is used for period-2 cycles. Color dark gray represents parameters that provide divergence of trajectories. Remaining colors are used for attractors consisting of more than a single point. (D): evaluation, for each parameters' coupling, of index $100 \cdot D$, where $D$ is defined in (13). Color dark blue represents parameters that provide diverging trajectories.

to "understand" the precise deterministic period-2 oscillation of the demand. In Figure 9 we consider the simulation reported in Figure 5 (B), in which the vertical shifting is now given by $\delta_{t}+h_{i}(\varepsilon)$, where $\delta_{t}$, is a sequence of independent normally distributed random variables with identical variance $\sigma=1$ and mean $\mu=0$. As we can see, it is actually impossible to recognize the cyclicity of the demand, even if the shock is small with respect to the perturbation size.

\section{Qualitative comparison with the empirical literature}

In this Section we aim at understanding if the assumptions at the basis of the present model are suitable to explain the emergence of several peculiar 


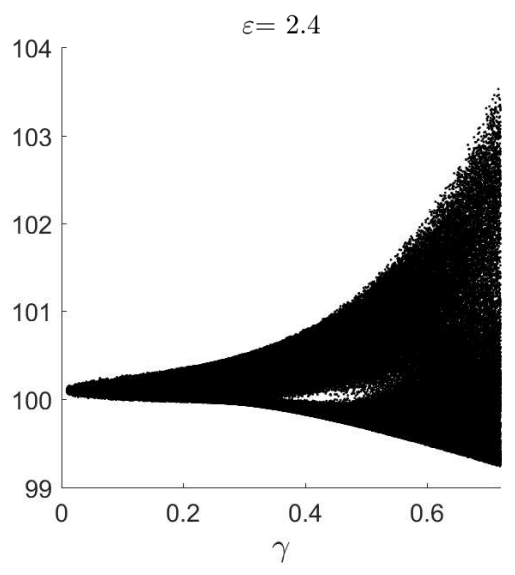

Figure 9: Bifurcation diagram for the same parameter configuration of the simulation reported in Figure $5(\mathrm{~B})$ with an additional random shock on the perturbation size.

characteristics in economic observables of actual markets. In particular, we are going to compare the qualitative characteristics of price series obtained through simulations and with those of actual price series. To this end, we again consider the case of a demand function which cyclically oscillates, so that such stylized framework resembles the situation in which the consumption of a good is affected by seasonality. As prototypical examples, we take into account the cases of agricultural or energy goods [29, 30]. As widely known, such goods are affected by price distributions that strongly deviate from normality, showing leptokurtic behavior, with positive skewness and significant volatility $[28]^{4}$. Also a significant positive autocorrelation is remarkable. A central debate in understanding price dynamics concerns the endogenous or exogenous cause of fluctuations, explaining them as either unstable and/or chaotic trajectories endogenously originated by the boundedly rational agents' forecasting errors or as the reaction of rationally behaving agents to real exogenous shocks. Usually, both extreme views have drawbacks in providing a satisfactory explanation of the emergence of the above mentioned stylized facts ([28]). In what follows, we test if the assumptions on which basis the model has been built together with

\footnotetext{
${ }^{4}$ We stress that in such work a cobweb approach is adopted to understand the agricultural price fluctuations. Concerning agricultural goods, as noticed in [29], "...the manner in which commodity prices are determined ranges from markets with near monopoly-like institutions, sometimes assisted by governmental regulation and intervention to approximations of the textbook definition of pricing under competitive conditions". Empirical estimations ([31]) concerning such markets highlight the existence of significant market powers. Similarly, several energy market are now deregulated, but also in this case "a road from state to private monopoly" ([30]) can be observed. All the authors remark that the above mentioned peculiarities in prices' distributions are common to such goods, quite independently of the way that prices are determined, even if deviation from normality is usually stronger under a competitive market assumption.
} 
the possibility of exogenous random shocks are suitable to foster the emergence of such qualitative aspects in the time series. The following results ${ }^{5}$ are obtained considering a cubic demand function and the same parameters used for the simulation reported in Figure $4(\mathrm{C})$. Finally, we slightly modify model (2) by introducing bounds to the maximum possible quantity variations, namely $q_{t+1}=q_{t}+\gamma \max \left\{\min \left\{\pi_{t+1}^{\prime}\left(q_{t}\right), \beta_{2}\right\}, \beta_{1}\right\}$, where $\beta_{2}>0$ and $\beta_{1}<0$ respectively represent the maximum possible increase and decrease of quantity. This has a clear economic motivation, as avoids the difference between the next period output decision $q_{t+1}$ and the current production decision $q_{t}$ to become unrealistically large. In real situations, firms are usually constrained in arbitrarily increasing or decreasing their production levels (see [32]). The use of similar bounding techniques is quite common in the modelling of analogous economic contexts, see for example $[33,34]$. We stress that, since the bounding mechanism is linear on $\left[\beta_{1}, \beta_{2}\right]$, both the analytical results of Section 3 and the simulations reported in Section 4 do not change as long as $q_{t} \in\left[\beta_{1}, \beta_{2}\right]$. In what follows we set $\beta_{1}=-8$ and $\beta_{2}=6$, which we checked to be sufficiently large so that all the results of the previous sections remain exactly the same. The bounding mechanism only acts when a sufficiently large shock occur and helps in preventing unrealistic divergence or negativity phenomena. Finally, we tested that the following results are robust on varying $\beta_{i}$ in a suitably large parameter region.

For each simulation, we compute the corresponding price series and we take into account 500 prices after an initial transient of 1000 time steps. In Figure 10 we report the results of kurtosis, skewness and percent relative volatility (the percent ratio between the standard deviation of the price distribution and the mean price) obtained considering different values of the agent's reaction speed $\gamma$ and of the standard deviation of the $\operatorname{shock}^{6}$. The values of each index are computed averaging 100 simulations, obtained with different sequences of shocks.

When $\gamma$ is small (below the lower solid line) and the dynamics are stable, results are in general not consistent with those empirical, with price distributions close to normality. Conversely, when $\gamma$ is sufficiently close to or larger than the parameter values which provide unstable dynamics, the price distributions become significantly non-normal, with excess kurtosis, positive skewness and increasing volatility. The values of kurtosis, skewness and volatility obtained with $\gamma$ belonging or close to the region of instability (namely, between or close to the two solid lines in Figure 10) are comparable to those reported in the above mentioned empirical literature [35, 29]. This suggests that the presence

\footnotetext{
${ }^{5}$ We stress that the aim of this section is not to model in detail any particular market, which is beyond of the scopes of the present contribution and would require to explicitly take into account economic, technological and institutional aspects of the market. Consistently with the whole approach, we keep the discussion at a theoretical level and we consider settings similar to those used in Section 4.

${ }^{6}$ We only considered $\gamma \in[0.4,1]$, as smaller values are slightly significant due to the increasingly slow convergence. However, if $\gamma$ is further decreased the results are comparable to those obtained for $\gamma \approx 0.4$.
} 
of the nonlinearity in the modelling framework is not sufficient to explain the non-normal distribution of prices, which arises and becomes significant only when the endogenous decision mechanism, grounded on the boundedly rational behavior of the agents, induces instability and consequent complex dynamics. The largest excess kurtosis as well as the most asymmetric distributions are mostly obtained in the region in which complex dynamics occur. We recall that in the deterministic bifurcation diagram (Figure $4(\mathrm{C})$ ) a bubbling phenomenon takes place, with a return to stability when $\gamma$ is sufficiently increased. A similar "bubbling effect" can be observed in Figures 10 (A,B,C,D), in which we can notice that both kurtosis and skewness decrease when the underlying deterministic dynamics simplify. Only volatility keeps on increasing as $\gamma$ increases, but this can be easily explained recalling that such index actually corresponds to the standard deviation of the price distribution, so it is more sensible to the size of the prices' distribution around their mean value than to the way they are distributed. If a random shock is present, as $\gamma$ increases, the difference between the largest and the smallest price increases (as for quantities in the bifurcation diagram reported in Figure 9), quite independently of the underlying deterministic dynamics. On this basis, we can conclude that volatility is also directly influenced by the agent's reactivity.

Concerning the size of the shock we take into account, we notice that it is relatively modest and it alone is unable to justify the extent of price fluctuations. Conversely, if we further increase the standard deviation of $\delta_{t}$, we have that the prices tend to become normally distributed, as the most significant effect is now the indeterministic one.

We remark that we checked the robustness of the previous results in several ways. For example, we modified the nonlinear demand function. We found that the essential aspect that allows reproducing qualitatively comparable results is the presence of a suitably significant nonlinearity in the function so that unstable and complex dynamics arise. Moreover, qualitatively comparable results and conclusions can be obtained considering also the other parameter settings used in Section 4. Indeed, in scenarios in which $\gamma$ has just a destabilizing role, kurtosis, skewness and volatility are only qualitatively increasing with $\gamma$. Finally, decreasing the size of the deterministic oscillation of the demand function, the previous peculiarities in the price series reduce or even disappear. This suggests that taking into account in the model the deterministic periodicity characterizing such markets is essential to correctly describe the price dynamics. All the previous considerations suggest that the fluctuating price dynamics of such kinds of markets can be explained as a joint effect of the boundedly rational nature of the economic agent, of a nonlinear economic setting and of an exogenous nondeterministic component. The reduced rationality of the monopolistic agent, in addition to being a realistic assumption $[2,1]$, fosters the emergence, in an economic setting characterized by nonlinearity, of endogenous, unstable complex dynamics. The setting becomes even more complex when it is also characterized by cyclicity, as analytically shown in Section 3, and the superposition of nondeterministic fluctuations leads price distributions into deviating from normality and exhibiting significant volatility. We notice that even if random shocks must 
be taken into account to obtain results comparable to those real, the dynamics are essentially driven by endogenous elements and allow avoiding specially-made assumptions on the shock distributions. This is also highlighted by the fact that a suitably small shock is sufficient to obtain prices whose distribution is very different from the normal distribution of the considered stochastic perturbation and that if a large shock is added, results become less significant as the shock distribution becomes dominant on the endogenous dynamics.

In the previous discussion we did not deal with one of the stylized facts we mentioned from the empirical literature, namely the positive autocorrelation of prices. In all the previous simulations, the first order autocorrelation coefficient is always significantly negative. However, this should not surprise too much, as this is a quite predictable consequence of the period-2 of deterministic oscillation we considered. At time $t+1$, any economic variable involved in the model must be negatively correlated with that at time $t$, since at two consecutive times the economic context actually upturns. This radically changes if we consider a "smoother" deterministic oscillation. If we consider the inverse demand function $p_{t}(q)=p_{0}(q)+\varepsilon \cos \left(\frac{2 \pi}{\omega} t\right), \omega \in \mathbb{N}$ we can model a symmetric oscillation with period $\omega$. Indeed, the analytical results derived in Section 3, which corresponds to $\omega=2$, are no more applicable to a general $\omega$, but model (2) can be applied without any change. Through simulations, we found that, as we increase the period of the oscillation, all the previous results concerning kurtosis, skewness and volatility are qualitatively confirmed and a significant positive autocorrelation emerges.

\section{Conclusions}

In this work we proposed a first model for a boundedly rational agent in a monopolistic market characterized by a time-varying demand function. The firm adjusts its production level on the basis of a gradient mechanism. Focusing on a simple kind of time-varying demand function, characterized by a cyclical recurrence with period two, we proved how the perturbation size and the agent's reactivity can affect the dynamics. On the basis of the profitability signal, the agent is able to recognize the kind of demand variability, and to adapt the production levels in a qualitatively correct way. However, the period-2 cycle toward which output trajectories converge does not consist, in general, of the profit maximizing output levels and depends on the agent's reactivity, and this moves away from the classical framework. Moreover, both perturbation size and agent's reaction speed can have a stabilizing effect on the resulting dynamics, unlike in the classical case. However, even if the model is very simple and does not require a precise knowledge by the agent of the underlying variability of the demand function, we show that the achieved profits are not so distant from the best profits. We also showed that the pursued approach can provide a qualitative justification of peculiarities characterizing economic observables in markets affected by seasonality, suggesting that deviation from normality of price distributions can be understood in terms of a nonlinear economic setting 
affected by a time-varying demand function, of a boundedly rational mechanism for the agents and of indeterministic perturbations.

This is a first attempt to investigate the effects of introducing time variability for the demand function. Indeed, several refinements of the proposed model or generalizations to different economic contexts can be studied. For example, considering again a monopolistic market, we can study models in which the agent is aware of the cyclicity of the demand, and tries to rely on it. Without this assumption, the mechanism adopted by the agent is, in some sense, not consistent with the underlying static optimization problem. Even if in the present contribution we aimed to provide a modelling approach suitable for any kind of time-varying demand and we studied the simplest kind of time variability as a prototypical example of more complex demand patterns, it is possible to model situations in which the agent is completely aware of the deterministic demand variations. This means that considering for instance a cyclically recurrent demand with period $n$, the agent, in order to choose the production level for time $t+1$, is sufficiently rational to use profitability signals from time $t+1-n$, knowing that the market is characterized by the same demand function at both times $t+1$ and $t+1-n$. The resulting model is more complicated than the present model from the mathematical point of view. First preliminary simulative results for a model encompassing a period-2 cyclicity show that however the stylized, qualitative behavior of the present model and of such an improved model are comparable.

Moreover, it would be interesting to understand how scenarios change when the (local) maximum point of the profit functions is no more unique. We also aim to investigate a time multiscale approach like that adopted in [19], to distinguish between the time levels of the learning processes and of the production decisions. Finally, generalizations to oligopolistic competitions are possible, also taking into account different kinds of heuristics.

\section{Acknowledgments}

The authors wish also to thank the two anonymous Referees for the helpful suggestions which allowed improving the quality of the paper.

\section{Appendix}

Proof of Proposition 1. The proof is straightforward. Let us consider function $G: \mathbb{R} \times[0, \hat{h}) \rightarrow \mathbb{R}$, defined by

$$
G(q, \varepsilon)=\pi_{0}^{\prime}(q)+h_{1}(\varepsilon) .
$$

Since $G\left(q^{*}, 0\right)=0$ and $\partial_{q} G(q, 0)=\pi_{0}^{\prime \prime}(q) \neq 0$, thanks to the implicit function theorem, equation $G(q, \varepsilon)=0$ defines a function $q_{1}(\varepsilon)$ on a suitable neighborhood $\left[0, \tilde{\varepsilon}_{1}\right)$ of $\varepsilon=0$. Thanks to the regularity assumptions on $\pi_{0}^{\prime}(q)$ and $h_{1}$, we have that $q_{1}(\varepsilon)$ is differentiable and $q_{1}(0)=q^{*}$. Moreover, we have $q_{1}^{\prime}(\varepsilon)=-h_{1}^{\prime}(\varepsilon) / \pi_{0}^{\prime \prime}\left(q_{1}(\varepsilon)\right)$, so, since $h_{1}^{\prime}>0$ and $\pi_{0}^{\prime \prime}<0$ we have $q_{1}^{\prime}(\varepsilon)>0$. 
Proceeding as before on function $G(q, \varepsilon)=\pi_{0}^{\prime}(q)+h_{2}(\varepsilon)$, we find a differentiable function $q_{2}(\varepsilon)$ implicitly defined on a suitable neighborhood $\left[0, \tilde{\varepsilon}_{2}\right)$ of $\varepsilon=0$, satisfying $q_{2}(0)=q^{*}$ and, since $q_{2}^{\prime}(\varepsilon)=-h_{2}^{\prime}(\varepsilon) / \pi_{0}^{\prime \prime}\left(q_{2}(\varepsilon)\right)$, recalling that $h_{2}^{\prime}<0$, for which we have $q_{2}^{\prime}<0$. Defining $q_{i}^{*}$ as the restrictions of $q_{i}$ on $\left[0, \varepsilon_{M}\right)=\min \left\{\tilde{\varepsilon}_{1}, \tilde{\varepsilon}_{2}\right\}$ concludes the proof.

Proof of Proposition 2. To study period-2 cycles of the non-autonomous model (5) we study steady states of the autonomous System (6). To this end, we consider map $G: \mathbb{R} \times[0, \hat{h}) \rightarrow \mathbb{R}$, defined by

$$
G\left(q_{2}, \varepsilon\right)=\pi_{0}^{\prime}\left(q_{2}\right)+\pi_{0}^{\prime}\left(q_{2}+\gamma\left(\pi_{0}^{\prime}\left(q_{2}\right)+h_{1}(\varepsilon)\right)\right)+h_{1}(\varepsilon)+h_{2}(\varepsilon),
$$

which, thanks to the regularity assumptions on functions $\pi_{0}^{\prime}$ and $h_{i}$, is continuously differentiable. Recalling (4a), we have $G\left(q^{*}, 0\right)=0$, while from (4b) and provided that $\gamma \neq-2 / \pi_{0}^{\prime \prime}\left(q^{*}\right)$ we have $\partial_{q_{2}} G\left(q^{*}, 0\right)=\pi_{0}^{\prime \prime}\left(q^{*}\right)+\pi_{0}^{\prime \prime}\left(q^{*}\right)(1+$ $\left.\gamma \pi_{0}^{\prime \prime}\left(q^{*}\right)\right) \neq 0$, so, from the implicit function theorem, we have that $G\left(q_{2}, \varepsilon\right)=0$ defines a continuously differentiable function $\hat{q}_{2}^{\gamma}(\varepsilon)$ for any $\gamma \in \Gamma=(0,1] \backslash$ $\left\{-2 / \pi_{0}^{\prime \prime}\left(q^{*}\right)\right\}$. For each $\gamma \in \Gamma$, let $I_{\gamma}=[0, \tilde{\varepsilon}(\gamma))$ be the largest possible open interval provided, for each $\gamma \neq-2 / \pi_{0}^{\prime \prime}\left(q^{*}\right)$, by the implicit function theorem. Let us introduce function $\hat{q}_{1}^{\gamma}: I_{\gamma} \rightarrow \mathbb{R}$ defined by $\hat{q}_{1}^{\gamma}(\varepsilon)=\hat{q}_{2}^{\gamma}(\varepsilon)+\gamma\left(\pi_{0}^{\prime}\left(\hat{q}_{2}^{\gamma}(\varepsilon)\right)+h_{1}(\varepsilon)\right)$.

Indeed, for each $\gamma \neq-2 / \pi_{0}^{\prime \prime}\left(q^{*}\right)$, both functions $\hat{q}_{i}^{\gamma}(\varepsilon)$ are continuous and differentiable with respect to $\varepsilon$. Moreover we have that $\left(\hat{q}_{1}^{\gamma}(\varepsilon), \hat{q}_{2}^{\gamma}(\varepsilon)\right)$ is a steady state of $(6)$. In fact, putting $\hat{q}_{2}^{\gamma}(\varepsilon)$ in the right hand side of each equation of (6), we indeed obtain $\hat{q}_{1}^{\gamma}(\varepsilon)$ in the first equation, and since the second equation can be rewritten as $q_{2, t+1}=q_{2, t}+\gamma G\left(q_{2, t}, \varepsilon\right)$ and from the definition of $\hat{q}_{2}^{\gamma}(\varepsilon)$, from the second equation we get $\hat{q}_{2}^{\gamma}(\varepsilon)$. This means that $\left\{\hat{q}_{1}^{\gamma}(\varepsilon), \hat{q}_{2}^{\gamma}(\varepsilon)\right\}$ is a 2 -cycle for (5). Thanks to the continuity of both functions $\hat{q}_{i}^{\gamma}(\varepsilon)$ we obtain point $(2)$, and this concludes the proof.

Proof of Proposition 3. Firstly we remark that, for each perturbation size for which $q_{i}^{*}(\varepsilon)$ are defined, the existence and uniqueness of $\bar{q}^{*}(\varepsilon)$ is a straightforward consequence of intermediate values theorem. As in the proof of Proposition 2, we study the steady states of System (6). To this end we consider map $G: \mathbb{R} \times \mathbb{R}^{+} \rightarrow \mathbb{R}$

$$
G\left(q_{2}, \gamma\right)=\pi_{0}^{\prime}\left(q_{2}\right)+\pi_{0}^{\prime}\left(q_{2}+\gamma\left(\pi_{0}^{\prime}\left(q_{2}\right)+h_{1}(\varepsilon)\right)\right)+h_{1}(\varepsilon)+h_{2}(\varepsilon),
$$

which, thanks to the regularity assumptions on $\pi_{0}^{\prime}$ and $h_{i}$, is differentiable. By the definition of $\bar{q}^{*}(\varepsilon)$, we have $G\left(\bar{q}^{*}(\varepsilon), 0\right)=2 \pi_{0}^{\prime}\left(\bar{q}^{*}(\varepsilon)\right)+h_{1}(\varepsilon)+h_{2}(\varepsilon)=0$. Moreover, since $\partial_{q_{2}} G\left(\bar{q}^{*}(\varepsilon), 0\right)=2 \pi_{0}^{\prime \prime}\left(\bar{q}^{*}(\varepsilon)\right) \neq 0$, thanks to the implicit function theorem we have that, for each $\varepsilon \in\left(0, \varepsilon_{M}\right)$, equation $G\left(q_{2}, \gamma\right)=0$ defines a continuously differentiable function $q_{2}^{\varepsilon}(\gamma)$. Then, for each $\varepsilon \in\left(0, \varepsilon_{M}\right)$, we can consider the largest interval $\tilde{I}_{\varepsilon}=[0, \tilde{\gamma}(\varepsilon))$ provided by the implicit function theorem and we can introduce function $q_{1}^{\varepsilon}: \tilde{I}_{\varepsilon} \rightarrow \mathbb{R}$ defined by $q_{1}^{\varepsilon}(\gamma)=q_{2}^{\varepsilon}(\gamma)+$ $\gamma\left(\pi_{0}^{\prime}\left(q_{2}^{\varepsilon}(\gamma)\right)+h_{1}(\varepsilon)\right)$.

Then, functions $\hat{q}_{i}^{\varepsilon}$ are simply defined as the restrictions of $q_{i}^{\varepsilon}$ on $I_{\varepsilon}=$ $\tilde{I}_{\varepsilon} \cap(0,1]$. Indeed, both functions $\hat{q}_{i}^{\varepsilon}$ are differentiable and, proceeding as in 
Proposition 2, $\left\{\hat{q}_{1}^{\varepsilon}(\gamma), \hat{q}_{2}^{\varepsilon}(\gamma)\right\}$ is a period-2 cycle for model (5). Thanks to the continuity of both functions $\hat{q}_{i}^{\varepsilon}(\gamma)$, from the previous considerations we immediately have point (2).

By means of implicit function theorem, we also have

$$
\begin{aligned}
\frac{d \hat{q}_{2}^{\varepsilon}(\gamma)}{d \gamma} & =-\frac{\frac{\partial G\left(\hat{q}_{2}^{\varepsilon}(\gamma), \gamma\right)}{\partial \gamma}}{\frac{\partial G\left(\hat{q}_{2}^{\varepsilon}(\gamma), \gamma\right)}{\partial q_{2}}} \\
& =-\frac{\pi_{0}^{\prime \prime}\left(\hat{q}_{2}^{\varepsilon}(\gamma)+\gamma\left(\pi_{0}^{\prime}\left(\hat{q}_{2}^{\varepsilon}(\gamma)\right)+h_{1}(\varepsilon)\right)\right) \cdot\left(\pi_{0}^{\prime}\left(\hat{q}_{2}^{\varepsilon}(\gamma)\right)+h_{1}(\varepsilon)\right)}{\pi_{0}^{\prime \prime}\left(\hat{q}_{2}^{\varepsilon}(\gamma)\right)+\pi_{0}^{\prime \prime}\left(\hat{q}_{2}^{\varepsilon}(\gamma)+\gamma\left(\pi_{0}^{\prime}\left(\hat{q}_{2}^{\varepsilon}(\gamma)\right)+h_{1}(\varepsilon)\right)\right) \cdot\left(1+\gamma \pi_{0}^{\prime \prime}\left(\hat{q}_{2}^{\varepsilon}(\gamma)\right)\right)}
\end{aligned}
$$

and

$$
\frac{d \hat{q}_{1}^{\varepsilon}(\gamma)}{d \gamma}=\frac{d \hat{q}_{2}^{\varepsilon}(\gamma)}{d \gamma}+\pi_{0}^{\prime}\left(\hat{q}_{2}^{\varepsilon}(\gamma)\right)+h_{1}(\varepsilon)+\gamma \pi_{0}^{\prime \prime}\left(\hat{q}_{2}^{\varepsilon}(\gamma)\right) \frac{d \hat{q}_{2}^{\varepsilon}(\gamma)}{d \gamma}
$$

We start focusing on $\lim _{\gamma \rightarrow 0^{+}} d \hat{q}_{2}^{\varepsilon}(\gamma) / d \gamma$. From the definition of $\hat{q}_{2}^{\varepsilon}(\gamma)$ and its regularity we have

$$
\lim _{\gamma \rightarrow 0^{+}} \pi_{0}^{\prime}\left(\hat{q}_{2}^{\varepsilon}(\gamma)\right)=\pi_{0}^{\prime}\left(\bar{q}^{*}(\varepsilon)\right)=-\frac{h_{1}(\varepsilon)+h_{2}(\varepsilon)}{2},
$$

where in the last equality we used the fact that $\bar{q}(\varepsilon)$ is a zero of $\bar{\pi}^{\prime}(q)$. Using (16), we have

$$
\lim _{\gamma \rightarrow 0^{+}} \pi_{0}^{\prime \prime}\left(\hat{q}_{2}^{\varepsilon}(\gamma)+\gamma\left(\pi_{0}^{\prime}\left(\hat{q}_{2}^{\varepsilon}(\gamma)\right)+h_{1}(\varepsilon)\right)\right) \cdot\left(\pi_{0}^{\prime}\left(\hat{q}_{2}^{\varepsilon}(\gamma)\right)+h_{1}(\varepsilon)\right)=\frac{h_{1}(\varepsilon)-h_{2}(\varepsilon)}{2} \pi_{0}^{\prime \prime}\left(\bar{q}^{*}(\varepsilon)\right)
$$

for the numerator of (14) and

$$
\lim _{\gamma \rightarrow 0^{+}} \pi_{0}^{\prime \prime}\left(\hat{q}_{2}^{\varepsilon}(\gamma)\right)+\pi_{0}^{\prime \prime}\left(\hat{q}_{2}^{\varepsilon}(\gamma)+\gamma\left(\pi_{0}^{\prime}\left(\hat{q}_{2}^{\varepsilon}(\gamma)\right)+\varepsilon\right)\right) \cdot\left(1+\gamma \pi_{0}^{\prime \prime}\left(\hat{q}_{2}^{\varepsilon}(\gamma)\right)\right)=2 \pi_{0}^{\prime \prime}\left(\bar{q}^{*}(\varepsilon)\right)
$$

for the denominator of (14) which proves

$$
\lim _{\gamma \rightarrow 0^{+}} \frac{d \hat{q}_{2}^{\varepsilon}(\gamma)}{d \gamma}=-\frac{h_{1}(\varepsilon)-h_{2}(\varepsilon)}{4}<0
$$

As a consequence, we have a neighborhood of $\gamma=0$ on which $d \hat{q}_{2}^{\varepsilon}(\gamma) / d \gamma$ is strictly negative. Moreover, the implicit function theorem guarantees that $\partial G / \partial q_{2}$ has constant sign on $I_{\varepsilon}$.

Similarly, proceeding as above, we have

$$
\lim _{\gamma \rightarrow 0^{+}} \frac{d \hat{q}_{2}^{\varepsilon}(\gamma)}{d \gamma}+\left(\pi_{0}^{\prime}\left(\hat{q}_{2}^{\varepsilon}(\gamma)\right)+h_{1}(\varepsilon)\right)+\gamma \pi_{0}^{\prime \prime}\left(\hat{q}_{2}^{\varepsilon}(\gamma)\right) \frac{d \hat{q}_{2}^{\varepsilon}(\gamma)}{d \gamma}=\frac{h_{1}(\varepsilon)-h_{2}(\varepsilon)}{4}>0,
$$

which concludes the proof of point (3) and guarantees that we have a neighborhood of $\gamma=0$ on which $d q_{1}^{*} / d \gamma$ is strictly positive. 
Combining the previous conclusions about the signs of $d \hat{q}_{2}^{\varepsilon}(\gamma) / d \gamma$ and $d q_{1}^{\varepsilon}(\gamma) / d \gamma$, we have a neighborhood of $\gamma=0$ on which $\hat{q}_{1}^{\varepsilon}(\gamma)>\bar{q}^{*}(\varepsilon)>\hat{q}_{2}^{\varepsilon}(\gamma)$. We can rewrite (14) as

$$
\frac{d \hat{q}_{2}^{\varepsilon}(\gamma)}{d \gamma}=-\frac{\pi_{0}^{\prime \prime}\left(\hat{q}_{1}^{\varepsilon}(\gamma)\right) \cdot\left(\frac{\hat{q}_{1}^{\varepsilon}(\gamma)-\hat{q}_{2}^{\varepsilon}(\gamma)}{\gamma}\right)}{\pi_{0}^{\prime \prime}\left(\hat{q}_{2}^{\varepsilon}(\gamma)\right)+\pi_{0}^{\prime \prime}\left(\hat{q}_{1}^{\varepsilon}(\gamma)\right) \cdot\left(1+\gamma \pi_{0}^{\prime \prime}\left(\hat{q}_{2}^{\varepsilon}(\gamma)\right)\right)},
$$

in which we used, both at the numerator and at the denominator, the steady state identity $\hat{q}_{1}^{\varepsilon}(\gamma)=\hat{q}_{2}^{\varepsilon}(\gamma)+\gamma\left(\pi_{0}^{\prime}\left(\hat{q}_{2}^{\varepsilon}(\gamma)\right)+h_{1}(\varepsilon)\right)$ coming from the first equation of (6). Using (18) in (15), after some algebraic manipulations we obtain

$$
\frac{d \hat{q}_{1}^{\varepsilon}(\gamma)}{d \gamma}=-\frac{\left(\frac{\hat{q}_{2}^{\varepsilon}(\gamma)-\hat{q}_{1}^{\varepsilon}(\gamma)}{\gamma}\right) \pi_{0}^{\prime \prime}\left(\hat{q}_{2}^{\varepsilon}(\gamma)\right)}{\pi_{0}^{\prime \prime}\left(\hat{q}_{2}^{\varepsilon}(\gamma)\right)+\pi_{0}^{\prime \prime}\left(\hat{q}_{1}^{\varepsilon}(\gamma)\right) \cdot\left(1+\gamma \pi_{0}^{\prime \prime}\left(\hat{q}_{2}^{\varepsilon}(\gamma)\right)\right)} .
$$

We notice that the denominator of both derivatives is $\partial G / \partial q_{2}$, which we already showed that is strictly negative on $I_{\varepsilon}$. Numerators of $d \hat{q}_{2}^{\varepsilon}(\gamma) / d \gamma$ and $d \hat{q}_{1}^{\varepsilon}(\gamma) / d \gamma$ must be respectively strictly negative and strictly positive. Otherwise, if for instance we had $d \hat{q}_{2}^{\varepsilon}(\gamma) / d \gamma=0$ at some $\gamma$, in (18) we would necessarily need $\hat{q}_{1}^{\varepsilon}(\gamma)-\hat{q}_{2}^{\varepsilon}(\gamma)=0$, since $\pi_{0}^{\prime \prime}<0$. Let $\gamma_{a}$ be the smallest value at which $\hat{q}_{1}^{\varepsilon}(\gamma)-\hat{q}_{2}^{\varepsilon}(\gamma)$ vanishes. Since $\hat{q}_{1}^{\varepsilon}(\gamma)$ is increasing and $\hat{q}_{2}^{\varepsilon}(\gamma)$ is decreasing in a neighborhood of $\gamma=0$, to have $\hat{q}_{1}^{\varepsilon}\left(\gamma_{a}\right)-\hat{q}_{2}^{\varepsilon}\left(\gamma_{a}\right)=0$ we necessarily need, at some $\gamma<\gamma_{a}$, that $\hat{q}_{1}^{\varepsilon}(\gamma)$ is decreasing and $\hat{q}_{2}^{\varepsilon}(\gamma)$ is increasing, which requires $d \hat{q}_{2}^{\varepsilon}(\gamma) / d \gamma=0$ at some $\gamma<\gamma_{a}$. This is a contradiction and concludes the proof.

Proof of Proposition 4. To prove the stability of the period-2 cycle of the nonautonomous model (5) we prove the stability of the steady state $\left(\hat{q}_{1}, \hat{q}_{2}\right)$ for the autonomous system (6), so we consider the Jacobian matrix $J\left(\hat{q}_{1}, \hat{q}_{2}\right)$ of System (6), evaluated at the steady state. Since the right hand sides of (6) only depends on $q_{2}$, its eigenvalues are 0 and $\lambda=j_{22}\left(\hat{q}_{1}, \hat{q}_{2}\right)$. A straightforward computation shows that the derivative of the right hand side of the second equation of (6) with respect to $q_{2}$ is

$$
1+\gamma \pi_{0}^{\prime \prime}\left(q_{2}\right)+\gamma \pi_{0}^{\prime \prime}\left(q_{2}+\gamma\left(\pi^{\prime}\left(q_{2}\right)+h_{1}(\varepsilon)\right)\right) \cdot\left(1+\gamma\left(\pi_{0}^{\prime}\left(q_{2}\right)\right)\right) .
$$

Noticing that $\hat{q}_{2}+\gamma\left(\pi^{\prime}\left(\hat{q}_{2}\right)+h_{1}(\varepsilon)\right)=\hat{q}_{1}$ and recalling that a steady state of $(6)$ is locally asymptotically stable provided that $|\lambda|<1$, we easily obtain (11).

Proof of Corollary 1. To show the possible emergence of the different scenarios, we consider marginal profit functions which are convex for $q<q_{F}$ and concave for $q>q_{F}$. Moreover, we assume that $q^{*}<q_{F}$, so that $\pi_{1}^{\prime}, \pi_{2}^{\prime}$ and $\bar{\pi}^{\prime}$ are convex at the equilibrium of the unperturbed problem. From point (4) of Proposition 3 we have that as $\gamma$ varies, $\hat{q}_{2}$ is decreasing and then $1+\gamma \pi^{\prime \prime}\left(\hat{q}_{2}\right)$ is decreasing, too, while $\hat{q}_{1}$ is increasing. We notice that $\pi^{\prime \prime}\left(\hat{q}_{1}\right)$ and $1+\gamma \pi^{\prime \prime}\left(\hat{q}_{1}\right)$ may be not monotonic with respect to $\gamma$. The first two scenarios obviously occur when, for 
instance $\left|1+\gamma \pi^{\prime \prime}\left(\hat{q}_{i}\right)\right|<1$ for any $\gamma$ (unconditionally stable) or when $1+\pi^{\prime \prime}\left(\hat{q}_{2}\right)<$ -1 but $1+\gamma \pi^{\prime \prime}\left(\hat{q}_{1}\right)>0$ for any $\gamma$ (destabilizing scenario). To prove the possible emergence of scenario (1c), in what follows we make reference to an illustrative situation reported in Figure 11. Marginal profit functions are illustrated in Figure $11(\mathrm{~A})$, in which the images of $\pi^{\prime}\left(\hat{q}_{1}\right)$ and $\pi^{\prime}\left(\hat{q}_{2}\right)$ as $\gamma$ increases are respectively represented in red and blue colors.

Recalling point (4) of Proposition 3, $\hat{q}_{2}$ is decreasing (blue line, Figure 11 (B)), so $\pi_{0}^{\prime \prime}\left(\hat{q}_{2}\right)$ is negative and decreasing, too (blue line, Figure $11(\mathrm{C})$ ). This means that $\left(1+\gamma \pi_{0}^{\prime \prime}\left(\hat{q}_{2}\right)\right)$ is positive for suitably small values of $\gamma$ and may become negative as $\gamma$ increases (blue line, Figure 11 (D)). Conversely, $\hat{q}_{1}$ is increasing (red line, Figure $11(\mathrm{~B}))$, so, since $\lim _{\gamma \rightarrow 0^{+}} \pi_{0}^{\prime \prime}\left(\hat{q}_{1}(\gamma)\right)>0 \pi_{1}^{\prime}\left(\hat{q}_{1}\right)$ is negative and increasing at least for sufficiently small values of $\gamma$ (red line, Figure $11(\mathrm{C}))$. If, for some $\gamma_{F}<1$, we have $\hat{q}_{1}=q_{F}$, then $\pi_{0}^{\prime \prime}\left(\hat{q}_{1}\right)$ is decreasing for $\gamma_{F}<\gamma \leq 1$ (Figure $11(\mathrm{D})$ ). If for example we have that $1+\gamma \pi_{0}^{\prime \prime}\left(\hat{q}_{1}\right)$ is positive for $\gamma \in\left(0, \gamma_{F}\right]$ and $1+\gamma_{F} \pi_{0}^{\prime \prime}\left(\hat{q}_{2}\right)<-1$, from the intermediate values Theorem and the previous monotonicity considerations, we have a unique $\gamma_{p d} \in\left(0, \gamma_{F}\right]$ at which $\left(1+\gamma \pi_{0}^{\prime \prime}\left(\hat{q}_{2}\right)\right) \cdot\left(1+\gamma \pi_{0}^{\prime \prime}\left(\hat{q}_{1}\right)\right)=-1$ and the 2-cycle becomes unstable through a period-doubling bifurcation (leftmost dotted green line, Figure 11 (D)). For $\gamma \in\left(\gamma_{F}, 1\right]$, we have that $1+\gamma \pi_{0}^{\prime \prime}\left(\hat{q}_{1}\right)$ is decreasing and if $\pi_{0}^{\prime \prime}\left(\hat{q}_{1}\right)$ is negative for $\gamma=1$, proceeding as before we have a unique $\gamma_{p h}$ at which $\left(1+\gamma \pi_{0}^{\prime \prime}\left(\hat{q}_{2}\right)\right) \cdot\left(1+\gamma \pi_{0}^{\prime \prime}\left(\hat{q}_{1}\right)\right)=-1$ and a period-halving bifurcation occurs (rightmost dotted green line, Figure 11 (D)), so that in a right neighborhood of $\gamma_{p h}$ the 2-cycle is again locally asymptotically stable.

\section{References}

\section{References}

[1] Baumol, W.J., Quandt, R.E.. Rules of thumb and optimally imperfect decisions. Amer Econ Rev 1964;54(2):23-46.

[2] Clower, R.W.. Some Theory of an Ignorant Monopolist. Econ J 1959;69(276):705-716.

[3] Robinson, J.. Economics of Imperfect Competition. London: Macmillan; 1933.

[4] Bischi, G. I., Naimzada, A.. Global Analysis of a Dynamic Duopoly Game with Bounded Rationality. In: Ann. Int. Soc. Dyn. Games. 2000, p. 361385 .

[5] Naimzada, A., Sbragia, L.. Oligopoly games with nonlinear demand and cost functions: Two boundedly rational adjustment processes. Chaos Solitons Fractals 2006;29:702-722.

[6] Anufriev, M., Kopanyi, D., Tuinstra, J.. Learning cycles in Bertrand competition with differentiated commodities and competing learning rules. J Econ Dyn Control 2013;37:2562-2581. 
[7] Cavalli, F., Naimzada, A.. A Cournot duopoly game with heterogeneous players: nonlinear dynamics of the gradient rule versus local monopolistic approach. Appl Math Comput 2014;249.

[8] Gori, L., Sodini, M.. A continuous time Cournot duopoly with delays. Chaos Solitons Fractals 2015;79:166-177.

[9] Cavalli, F., Naimzada, A., Tramontana, F.. Nonlinear dynamics and global analysis of a heterogeneous Cournot duopoly with a local monopolistic approach versus a gradient rule with endogenous reactivity. Commun Nonlinear Sci Numer Simul 2015;23(1-3):245-262.

[10] Puu, T.. The chaotic monopolist. Chaos Solitons Fractals 1995;5(1):35-44.

[11] Naimzada, A., Ricchiuti, G.. Complex dynamics in a monopoly with a rule of thumb. Appl Math Comput 2008;203(2):921-925.

[12] Askar, S.. On complex dynamics of monopoly market. Econ Model 2013;31(1):586-589.

[13] Matsumoto, A., Szidarovszky, F.. Complex dynamics of monopolies with gradient adjustment. Econ Model 2014;42:220-229.

[14] Matsumoto, A., Szidarovszky, F.. Learning monopolies with delayed feedback on price expectations. Commun Nonlinear Sci Numer Simul 2015;28(13):151-165.

[15] Elsadany, A.A., Awad, A.M.. Dynamical analysis of a delayed monopoly game with a log-concave demand function. Oper Res Lett 2016;44:33-38.

[16] Cavalli, F., Naimzada, A.. Effect of price elasticity of demand in monopolies with gradient adjustment. Chaos Solitons Fractals 2015;76.

[17] Matsumoto, A., Szidarovszky, F.. Nonlinear delay monopoly with bounded rationality. Chaos Solitons Fractals 2012;45(4):507-519.

[18] Matsumoto, A., Szidarovszky, F.. Dynamic monopoly with multiple continuously distributed time delays. Math Comp Simul 2015;108:99-118.

[19] Cavalli, F., Naimzada, A.. A multiscale time model with piecewise constant argument for a boundedly rational monopolist. J Differ Equations Appl 2016;in press,.

[20] Gori, L., Guerrini, L., Sodini, M.. Different Modelling Approaches for Time Lags in a Monopoly. In: Essays Econ. Dyn. 2016, p. 81-98.

[21] Cavalli, F., Naimzada, A., Parisio, L.. A cobweb model for electricity markets. In: IEEE Conf. Proc. 2015,.

[22] Wray, M.. Seasonal Demand and Uncertainty in Consumer Goods Industries-Some Case Study Data. J Ind Econ 1958;7:44-65. 
[23] Grant, J. H., Lambert, D.M., Foster, K.. A Seasonal inverse almost ideal demand system for North American fresh tomatoes. Can J Agric Econ 2010;58:215-234.

[24] Soysal, G.P., Krishnamurthi, L.. Demand dynamics in the seasonal goods industry: An empirical analysis. Mark Sci 2012;31:293-316.

[25] Grinfeld, M., Knight, P. A., Lamba, H.. On the periodically perturbed logistic equation. J Phys A Math Gen 1996;29:8035-8040.

[26] AlSharawi Z., Angelos J., Elaydi S., Rakesh, L.. An extension of Sharkovsky's theorem to periodic difference equations. J Math Anal Appl 2006;316:128-141.

[27] Elaydi, S., Luis, R., Oliveira, H.. Local bifurcation in one-dimensional nonautonomous periodic difference equation. Int $\mathrm{J}$ Bifurc Chaos 2013;23:1350049.

[28] Gouel, C.. Agricultural price instability: a survey of competing explanations and remedies. J Econ Surveys 2012;26:129-156.

[29] Tomek, W., Kaiser, H.. Agricultural Product Prices. Cornell University Press; 2014.

[30] Weron, R., Simonsen, I., Wilman, P.. Modeling highly volatile and seasonal markets: evidence from the nord pool electricity market. In: Takayasu, H., editor. The Application of Econophysics. 2004, p. 182-191. Proceedings of the Second Nikkei Econophysics Symposium.

[31] Felis, A., Garrido, A.. Market power dynamics and price volatility in markets of fresh fruits and vegetables. Working Paper 7; Research Centre for the Management of Agricultural and Environmental Risks (CEIGRAM), Technical University of Madrid, Spain; 2015. ULYSSES project, EU 7th Framework Programme.

[32] Sterman, J.. Business Dynamics: Systems Thinking and Modeling for a Complex World. McGraw-Hill; 2000.

[33] Du, J.G., Fan, Y.Q., Sheng, Z.H., Hou, Y.Z.. Dynamics analysis and chaos control of a duopoly game with heterogeneous players and output limiter. Economic Modelling 2013;33:507-516.

[34] Cavalli, F., Naimzada, A., Pireddu, M.. Heterogeneity and the (de)stabilizing role of rationality. Chaos Solitons Fractals 2015;79:226-244.

[35] Deaton, A., Laroque, G.. On the behaviour of commodity prices. Rev Econ Stud 1992;. 


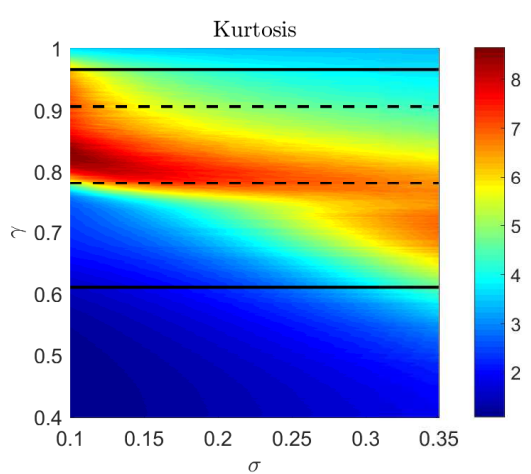

(A)

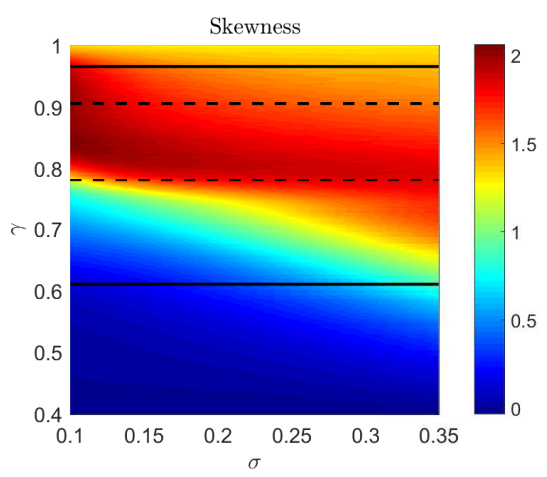

(C)

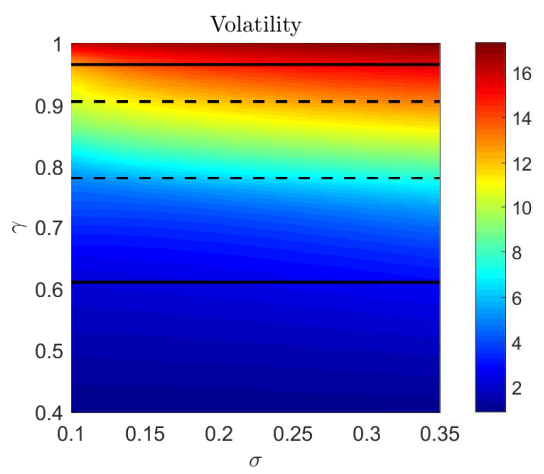

(E)

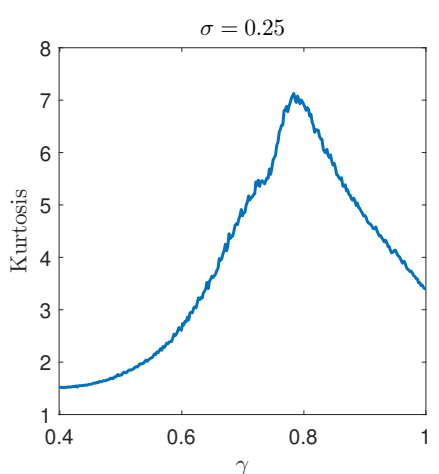

(B)

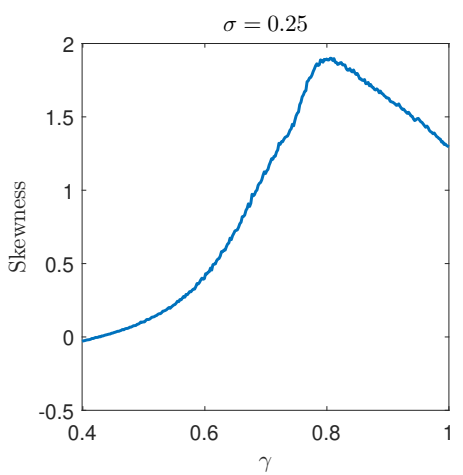

(D)

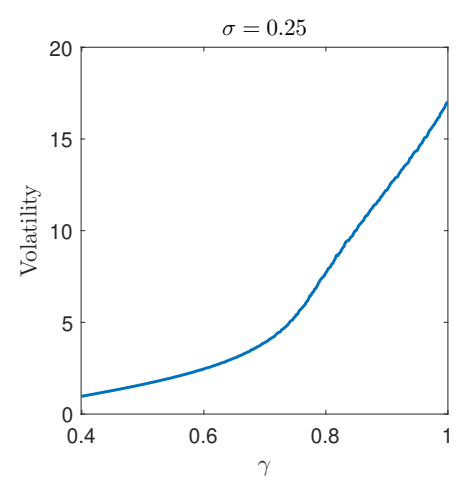

$(\mathrm{F})$

Figure 10: Plot of the kurtosis (A,B), skewness (C,D) and volatility (E,F) for the distributions of prices. Left column: figures are obtained on varying the agent's reactivity $\gamma$ and for different values of the standard deviation $\sigma$ of the stochastic shock. Horizontal solid lines represent the region inside which the period-2 cycle becomes unstable. Dashed lines represent the region inside which complex chaotic dynamics arise. Right column: plots corresponding to $\sigma=0.25$. 


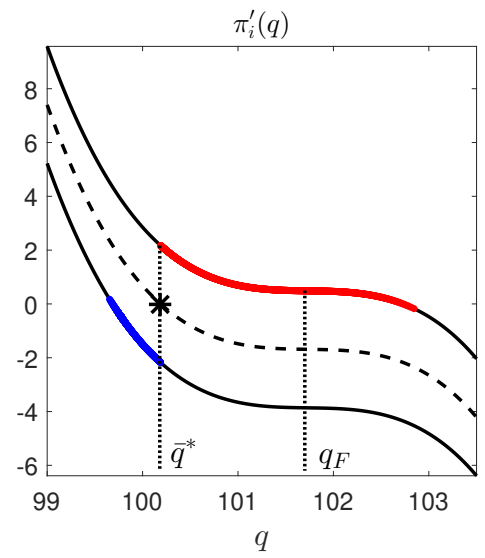

(A)

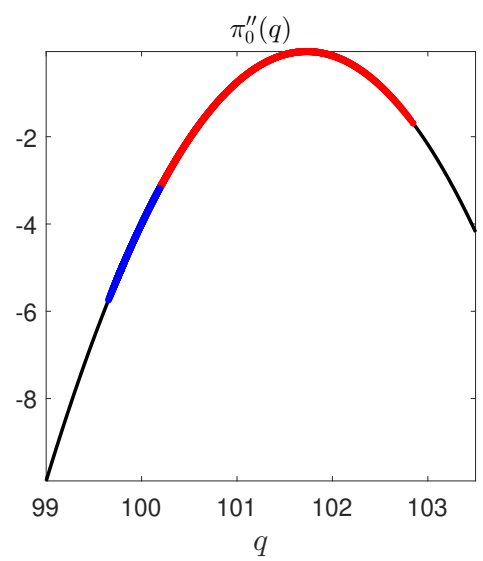

(C)

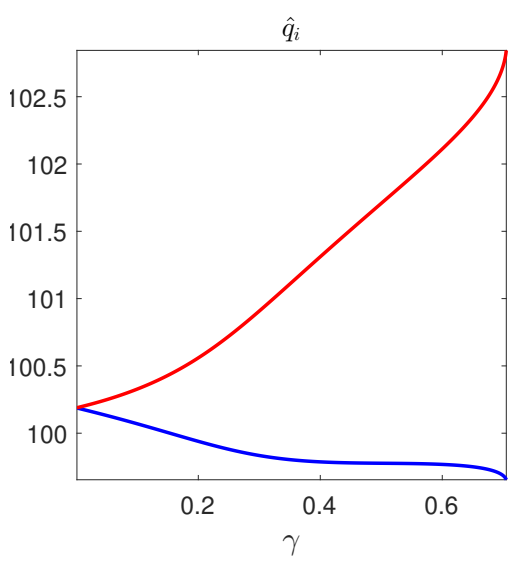

(B)

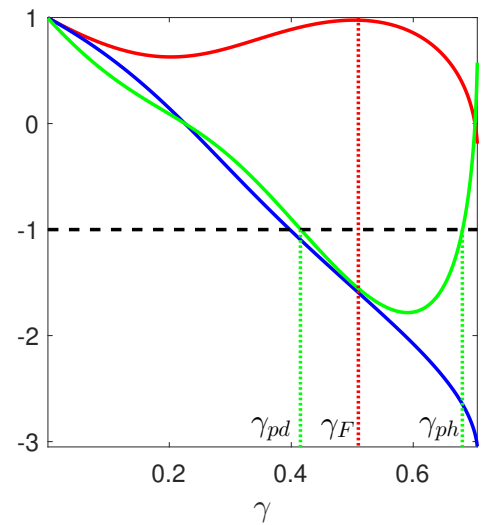

(D)

Figure 11: (A): marginal profit functions $\pi_{0}^{\prime}$ (dashed line) and $\pi_{1}^{\prime}, \pi_{2}^{\prime}$ (respectively upper and lower solid lines). Red (resp. blue) color is used for the images of the increasing (resp. decreasing) sequence of values $\hat{q}_{1}^{\varepsilon}(\gamma)$ (resp. $\hat{q}_{2}^{\varepsilon}(\gamma)$ ) as $\gamma$ increases. (B): Plots of $\hat{q}_{1}^{\varepsilon}(\gamma)$ (red color) and $\hat{q}_{2}^{\varepsilon}(\gamma)$ (blue color). (C): derivative of the marginal profit function, with superimposed red and blue points representing the images of the sequence of values $\pi_{0}^{\prime \prime}\left(\hat{q}_{1}^{\varepsilon}(\gamma)\right)\left(\operatorname{resp} \cdot \pi_{0}^{\prime \prime}\left(\hat{q}_{2}^{\varepsilon}(\gamma)\right)\right)$ as $\gamma$ increases. (D): Red line represents $1+\gamma \pi_{0}^{\prime}\left(\hat{q}_{1}^{\varepsilon}(\gamma)\right)$, blue line line represents $1+\gamma \pi_{0}^{\prime}\left(\hat{q}_{2}^{\varepsilon}(\gamma)\right)$, green line represents stability condition (11) as $\gamma$ increases. 